\title{
WestVirginiaUniversity
}

THE RESEARCH REPOSITORY @ WVU

Graduate Theses, Dissertations, and Problem Reports

2013

\section{Continuities on Subspaces}

Timothy James Glatzer

West Virginia University

Follow this and additional works at: https://researchrepository.wvu.edu/etd

\section{Recommended Citation}

Glatzer, Timothy James, "Continuities on Subspaces" (2013). Graduate Theses, Dissertations, and Problem Reports. 384.

https://researchrepository.wvu.edu/etd/384

This Dissertation is protected by copyright and/or related rights. It has been brought to you by the The Research Repository @ WVU with permission from the rights-holder(s). You are free to use this Dissertation in any way that is permitted by the copyright and related rights legislation that applies to your use. For other uses you must obtain permission from the rights-holder(s) directly, unless additional rights are indicated by a Creative Commons license in the record and/ or on the work itself. This Dissertation has been accepted for inclusion in WVU Graduate Theses, Dissertations, and Problem Reports collection by an authorized administrator of The Research Repository @ WVU.

For more information, please contact researchrepository@mail.wvu.edu. 


\title{
Continuities on Subspaces
}

\author{
Timothy James Glatzer, M.S. \\ Dissertation submitted \\ to the Eberly College of Arts and Sciences \\ at West Virginia University \\ in partial fulfillment of the requirements for the degree of \\ Doctor of Philosophy in \\ Mathematics
}

Krzysztof Ciesielski, Ph.D., Chair

Jerzy Wojciechowski, Ph.D.

John L. Goldwasser, Ph.D.

Edgar Fuller, Ph.D.

Robert Mnatsakanov, Ph.D.

Department of Mathematics

Morgantown, West Virginia

2013

Keywords: separate continuity, linear continuity, discontinuity

sets

Copyright 2013 Timothy J. Glatzer 


\title{
Abstract
}

\section{Continuities on Subspaces}

\author{
Timothy J. Glatzer
}

We define a generalized continuity by declaring that for any family $\mathcal{S}$ of subsets of a topological space $X$, a function $f: X \rightarrow Y$ is $\mathcal{S}$-continuous if for each $S \in \mathcal{S}$, the function $f\lceil S: S \rightarrow Y$ is continuous. This is easily seen to generalize such well known concepts as separate continuity and linear continuity. Using this definition as a way to unify several disparate results, we attempt to create a theory of $\mathcal{S}$-continuity. As a part of this program, we give constructions for $\mathcal{S}$-continuous functions for several natural classes $\mathcal{S}$, describe the sets of discontinuities of such functions (characterizing several classes), and discuss the regularity of such functions. 


\section{Acknowledgements}

The time during which this document took form has been vicissitudinous and challenging. I would be deeply remiss to not attempt to express my gratitude to many people, however, I freely acknowledge that a few feeble words will not begin to repay the debts I owe.

I begin by extending my gratitude to my wife, Wendy, who has been an unwaivering source of love and support during these years and without whom I would be quite lost.

Thanks are due to my parents, Jim and Cheryl Glatzer, whose love, guidance, assistance, and wisdom have seen me through over thirty years and inspired me to always learn and strive to be my best. I also extend my thanks to my step mother, Shelley for her willingness to assume a difficult role and my Aunt Jeanette who always made sure I did my math homework.

I thank my committee: Dr. Wojciechowski, Dr. Fuller, Dr. Goldwasser, and Dr. Mnatsakanov and most especially my advisor, Dr. Krzysztof Ciesielski without whose encouragement, advice, assistance, and (infinite) patience this paper would be much smaller, written on the backs of shopping reciepts, and mostly wrong.

Thanks are also due to Dr. Piotrowski of Youngstown State University, 
who suggested many of these problems to us.

I thank West Virginia University for providing me years of support and first rate education.

Lastly, I thank God for providing me the ability to do this work and for ensuring that I should always have a meaningful task before me.

My time as a graduate student was bookmarked by losses: my maternal grandfather passed on as I began my graduate studies; my grandmother joined her husband during my last year of graduate studies. All of my grandparents made it clear to me, throughout their lives, that they fully believed I would accomplish something great. Their enthusiasm and love has been a comfort and inspiration to me. Therefore, I dedicate this effort to their memories. 


\section{Contents}

1 Preliminaries 2

1.1 Introduction . . . . . . . . . . . . . . . . 2

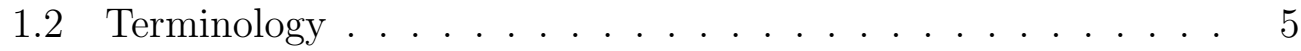

1.3 History . . . . . . . . . . . . . . . . . . 10

2 Basic Results 19

2.1 Introduction . . . . . . . . . . . . . . . . . . . . . 19

2.2 Basic Theorems . . . . . . . . . . . . . . . . . . . . . 21

2.3 A Basic Lemma . . . . . . . . . . . . . . . . . . . . 26

$3 \mathfrak{C}\left(\mathcal{D}^{2}\right)$-continuous Functions with Large Discontinuity Sets 28

3.1 Introduction . . . . . . . . . . . . . . . . . 28

3.2 The First Construction . . . . . . . . . . . . . . . . . . . . . . . 29

3.3 The Second Construction . . . . . . . . . . . . . . . . 35

3.4 Discussion . . . . . . . . . . . . . . . . . . . . . . 39

4 Sufficient Conditions for Membership in $\mathcal{D}_{1, n}$. 41

4.1 Introduction . . . . . . . . . . . . . . . . . 41

4.2 A Sufficient Condition for Membership in $\mathcal{D}_{1, n} \ldots \ldots \ldots$ 
4.3 A Sufficient Condition for $\mathbb{R}^{2}$. . . . . . . . . . . . . 50

4.4 Discussion . . . . . . . . . . . . . . . . . . 52

5 Characterizations of Discontinuity Sets 53

5.1 Introduction . . . . . . . . . . . . . . . 53

5.2 Characterization of the Sets $\mathcal{D}_{k, n}^{+} \ldots \ldots \ldots 55$

$5.3 \mathcal{F}_{k}$-continuous Functions and Some Structure of the Sets in $\mathcal{D}_{k, n} 56$

5.4 A Characterization of $D_{k, n}$ when $k \geq \frac{n}{2}$. . . . . . . . 60

5.5 Proof of Theorem 5.2.1 . . . . . . . . . . . . 61

5.6 Proof of Theorem 5.3.3 . . . . . . . . . . . . . . 63

5.7 Proof of Theorem 5.4.3 . . . . . . . . . . . . 67

5.8 A Note on Baire Class . . . . . . . . . . . . . . 77

6 Open Questions $\quad 79$ 


\section{Chapter 1}

\section{Preliminaries}

\subsection{Introduction}

The concept of limit, and hence of continuity, is more complicated for functions defined on $\mathbb{R}^{n}$ when $n>1$ than it is for functions defined on $\mathbb{R}$. For example, in $\mathbb{R}^{2}$, the plurality of routes to the point $\left\langle x_{0}, y_{0}\right\rangle$ complicates the calculation of the expression $\lim _{\langle x, y\rangle \rightarrow\left\langle x_{0}, y_{0}\right\rangle} f(x, y)$. Usually, the way that this phenomenon is demonstrated to students is through a function like the following.

$$
f(x, y)= \begin{cases}\frac{2 x y}{x^{2}+y^{2}} & \text { if }\langle x, y\rangle \neq\langle 0,0\rangle \\ 0 & \text { if }\langle x, y\rangle=\langle 0,0\rangle .\end{cases}
$$

This function is discontinuous at the origin. For every neighborhood $U$ of $\langle 0,0\rangle$, there is a point of the diagonal $D=\{\langle x, x\rangle: x \in \mathbb{R}\}$ in $U$, and at this point, $f$ takes the value 1 . However, every such neighborhood contains $\langle 0,0\rangle$ where the function takes the value 0 . Hence, the oscillation of $f$ at $\langle 0,0\rangle$ is 
equal to 1 . However, for any fixed $x_{0} \in \mathbb{R}$, the function $f_{x_{0}}(y) \stackrel{\text { def }}{=} f\left(x_{0}, y\right)$ is continuous. Also, for every fixed $y_{0}$, the function $f^{y_{0}}(x) \stackrel{\text { def }}{=} f\left(x, y_{0}\right)$ is continuous. We frequently use the notation $f\left(\cdot, y_{0}\right)$ for the function $f^{y_{0}}(x)$ and $f\left(x_{0}, \cdot\right)$ for the function $f_{x_{0}}(y)$. So, the function $f(x, y)$ has the property that $f(\cdot, y)$ and $f(x, \cdot)$ are continuous, for each $x$ and $y$, although $f(x, y)$ is discontinuous. In general, a function $f: \mathbb{R}^{n} \rightarrow \mathbb{R}$ is called separately continuous if for each $i$ between 1 and $n$ and each fixed $\left(x_{1}, \ldots, x_{i-1}, x_{i+1}, \ldots x_{n}\right)$ the function $f\left(x_{1}, \ldots, x_{i-1}, \cdot, x_{i+1}, \ldots, x_{n}\right)$ is continuous.

Separate continuity is here defined rather algebraically - as the continuity of the function "in each variable." However, the geometric interpretation of this concept is what best allows us generalize this concept further. Geometrically, the content of separate continuity is that the function is continuous when restricted to any line parallel to any of the coordinate axes. The fact that discontinuous separately continuous functions exist, coupled with this geometric description invites the question "Are there discontinuous functions whose restriction to any line is continuous?" Again, the answer has been known for quite some time. Consider the following function, first published in 1884, in a calculus text by Gennochi and Peano [21].

$$
f(x, y)= \begin{cases}\frac{x y^{2}}{x^{2}+y^{4}} & \text { if }\langle x, y\rangle \neq\langle 0,0\rangle \\ 0 & \text { if }\langle x, y\rangle=\langle 0,0\rangle\end{cases}
$$

Clearly, the only discontinuity can be at the origin. Notice, that for any line 
of the form $y=c x$, we have the following

$$
\lim _{x \rightarrow 0} f(x, c x)=\lim _{x \rightarrow 0} \frac{c x^{3}}{x^{2}+c^{4} x^{4}}=0
$$

Also, if we look at the $y$-axis, we see that

$$
\lim _{y \rightarrow 0} f(0, y)=\lim _{y \rightarrow 0} \frac{0}{0+y^{4}}=\lim _{y \rightarrow 0} 0=0
$$

Thus, every restriction of the function to a line is continuous. However, note that every neighborhood of the origin contains a point of the parabola $y=x^{2}$. Evaluating the function at such a point, we have

$$
f\left(y^{2}, y\right)=\frac{y^{4}}{2 y^{4}}=\frac{1}{2} \neq 0
$$

Hence, the function has positive oscillation at the origin, and is therefore discontinuous there. Functions, such as the function of (1.2) whose restriction to any line is continuous are called linearly continuous. Separate continuity and linear continuity are just two examples of what we call restriction continuities. For any family $\mathcal{S}$ of subsets of our domain, $X$, we can define a restriction continuity by decreeing that a function $f: X \rightarrow Y$ is $\mathcal{S}$-continuous if, and only i,f the restriction $f\lceil S$ is continuous for every $S \in \mathcal{S}$.

There is a wealth of past results on related subjects, however, many of these results did not treat restriction continuities in general as an object of study. One of the purposes of this document is to create a formal framework in which these generalized continuities can be studied. To this end, we discuss ways in which several seemingly disparate results are related. How- 
ever, the main raison d'etre of this document is to answer basic questions related to restriction continuities. The questions tend to fall into several broad categories. In the main, we shall focus on describing the sets of discontinuity of an $\mathcal{S}$-continuous function for various $\mathcal{S}$, determining how the different classes of $\mathcal{S}$-continuity relate to one another, and the construction of functions satisfying these continuities.

\subsection{Terminology}

Our terminology and notation choices are fairly standard, with the exception of our somewhat unusual notation for balls in metric spaces. We denote by $B(x, r)$ the open ball of radius $r$ centered at $x$. For the closure of the ball $B(x, r)$, we write $B[x, r]$. We denotre the open and closed balls of radius $r$ centered at the origin as $B(r)$ and $B[r]$ respectively. The metric in $\mathbb{R}^{n}$ will usually be denoted by $\|x-y\|$ if $n>1$. Furthermore, in any metric space $Z$, for any point $x \in Z$ and subset $S \subset Z$, we may define the quantity $\operatorname{dist}(x, S)=\inf \{d(x, s): s \in S\}$.

We will follow the set theoretic terminology used by Ciesielski [7], our topological terminology will follow Engelking [18], and our analytic terminology will follow Royden [42], Oxtoby [35], and Falconer [20]. In particular, following [7], we identify a function with its graph and denote the first infinite ordinal by $\omega$. Given a function $f: X \rightarrow Y$ between topological spaces $X$ and $Y$, we denote the restriction of $f$ to a subset $S$ of $X$ by $f \uparrow S$. The restriction of $f$ to $S$ is the function $f\lceil S: S \rightarrow Y$ where $(f\lceil S)(x)=f(x)$ for all $x \in S$. The support of a function $f$, denoted $\operatorname{supp}(f)$ is the closure of the 
set $\{x: f(x) \neq 0\}$.

The notation $\mathcal{C}^{n}(X, Y)$ will denote the set of all $n$-times continuously differentiable functions from $X$ to $Y$, where $X$ and $Y$ possess sufficient structure to allow "differentiability" to be defined, e.g. they may be subsets of $\mathbb{R}^{n}$. If $Y=\mathbb{R}$, then we suppress its mention. If $Y=\mathbb{R}$ and $X$ is obvious from the context, we supress the argument entirely and write $\mathcal{C}^{n}$. The notation " $\mathcal{C}^{n}(X, Y)$ " will refer to the set of $n$-times continuously differentiable functions, when we allow the $n$-th derivative to assume infinite values. Likewise $D^{n}(X, Y)$ will be the set of all functions with $n$ derivatives. The set of real analytic (i.e. possessing convergent real Maclaurin series) functions from $X$ to $Y$ will be denoted $\mathcal{A}(X, Y)$. Since, we identify a function with its graph, the notation $\mathcal{C}^{n}\left(\mathbb{R}^{n}\right)$ can refer to the set of all $n$-times continuously differentiable real-valued functions on $\mathbb{R}^{n}$, or the set of all such graphs of functions sitting in $\mathbb{R}^{n+1}$. Similar remarks apply to the other classes of functions. The set of all Lipschitz functions defined on a space $X$ is denoted $\operatorname{Lip}(X)$.

A curve is understood as the range of a continuous injection $\alpha: J \rightarrow \mathbb{R}^{n}$, where $J$ is an interval and $\alpha=\left\langle h_{1}, h_{2}, \ldots, h_{n}\right\rangle$. We will call a curve $\mathcal{C}^{1}$ if $h_{i}$ is $\mathcal{C}^{1}$ for all $1 \leq i \leq n$ and we shall call a curve smooth if it is $\mathcal{C}^{1}$ and $\left\langle h_{1}^{\prime}(t), h_{2}^{\prime}(t), \ldots h_{n}^{\prime}(t)\right\rangle \neq\langle 0,0, \ldots, 0\rangle$ for every $t \in J$. Furthermore, our curve will be said to be $\mathcal{C}^{n}$ if it is $\mathcal{C}^{1}$ and its coordinate functions are $n$ times continuously differentiable. Similarly, a curve is $D^{n}$ (for $n>1$ ) if it is $\mathcal{C}^{1}$ and its coordinate functions are $n$-times differentiable. We will also have occasion to discuss analytic curves; $\mathcal{C}^{1}$ curves whose component functions are real analytic. The class of all $\mathcal{C}^{n}$ curves (respectively $D^{n}$ curves or analytic curves) is denoted $\mathfrak{C}\left(\mathcal{C}^{n}\right)$, (repectively $\mathfrak{C}\left(D^{n}\right)$ or $\left.\mathfrak{C}(\mathcal{A})\right)$. 
We use the above sets to describe various restriction continuities. For any of the classes of curves, $\mathfrak{C}$, we may study $\mathfrak{C}$-continuous functions. We will also study $\mathcal{S}$-cotninuous function for various affine sets $\mathcal{S}$. For functions $f: \mathbb{R}^{n} \rightarrow \mathbb{R}$, we say that $f$ is separately continuous if $f \nmid L$ is continuous for every line $L$ parallel to some coordinate axis. If $f\lceil L$ is continuous for every line, we say that $f$ is linearly continuous. Besides lines, we are also interested in the case when $\mathcal{S}$ is a class of flats. A $k$-flat in $\mathbb{R}^{n}$ is a translate of a $k$-dimensional subspace. If $f \nmid F$ is continuous for every $k$-flat $F$, then we say that $f$ is $k$-flat continuous or $\mathcal{F}_{k}$-continuous. If $f\lceil F$ for every $k$-flat $F$ parallel to some set of coordinate axes, then we say that $f$ is $\mathcal{F}_{k}^{+}$-continuous.

For any function $f: X \rightarrow Y$ for any topological spaces $X$ and $Y$, we denote by $D(f)$, the set $\{x: f$ has a discontinuity at $x\} . D(f)$ is sometimes called the discontinuity set of $f$. We also denote by $C(f)$ the set $\{x: f$ is continuous at $x\}$. This set is frequently called the continuity set of $f$.

A major part of our investigations shall be focused on the study of sets of discontinuity of various classes of functions. The collections of sets under consideration will be denoted as follows: $\mathcal{D}_{1, n} \stackrel{\text { def }}{=}\left\{D(f): f: \mathbb{R}^{n} \rightarrow \mathbb{R}\right.$ is linearly continuous $\}, D_{1, n}^{+} \stackrel{\text { def }}{=}\left\{D(f): f: \mathbb{R}^{n} \rightarrow \mathbb{R}\right.$ is separately continuous $\}$. $\mathcal{D}_{k, n} \stackrel{\text { def }}{=}\left\{D(f): f: \mathbb{R}^{n} \rightarrow \mathbb{R}\right.$ is $\mathcal{F}_{k}$-continuous $\}, \mathcal{D}_{k, n}^{+} \stackrel{\text { def }}{=}\left\{D(f): f: \mathbb{R}^{n} \rightarrow \mathbb{R}\right.$ is a $k$-continuous function $\}$. For general collections $\mathcal{S}$ of subsets of $X$, we use $\mathcal{D}_{\mathcal{S}} \stackrel{\text { def }}{=}\{D(f): f: X \rightarrow Y$ is $\mathcal{S}$-continuous $\}$.

To discuss families of $\mathcal{S}$-continuous functions, we use the following notation. For any family $\mathcal{S}$ of subsets of a space $X$, we denote the set of $\mathcal{S}$-continuous functions by $\mathcal{C}_{\mathcal{S}}(X)$. If $\mathcal{S}$ is the set of all lines (respectively $k$ flats) the set of $\mathcal{S}$-continuous functions on $\mathbb{R}^{n}$ is denoted by $\mathcal{C}_{1}^{n}$. (respectively 
$\mathcal{C}_{k}^{n}$.)

Furthermore, we shall say that a function $f: X \rightarrow Y$ (for any topological spaces $X$ and $Y$ ) is Darboux if for every connected subset $C$ of $X, f[C]$ is a connected subset of $Y$. The function $f$ will be said to be of the first Baire class (or Baire class 1), a statement denoted $f \in \mathcal{B}_{1}$, if $f(x)=\lim _{n \rightarrow \infty} f_{n}(x)$ where each $f_{n}$ is continuous. In general, we say that $f$ is of the $n^{\text {th }}$ Baire class (denoted $f \in \mathcal{B}_{n}$ ) if $f(x)=\lim _{n \rightarrow \infty} f_{n}(x)$ and each $f_{n}$ is of Baire class less than $n$. It is worth noting that all derivatives are Darboux and of the first Baire class, however, the intersection of these two classes contains more than just derivatives. See, for example, [5], for details.

A set which is a countable union of closed sets is called an $F_{\sigma}$ set and a set which is a countable intersection of open sets is called a $G_{\delta}$ set. A countable intersection of $F_{\sigma}$ sets is called an $F_{\sigma \delta}$ set, while a set which is a countable union of $G_{\delta}$ sets is called a $G_{\delta \sigma}$. For more on these classes, see [7, Section 6.2].

We denote $n$-dimensional Lebesgue measure by $\lambda_{n}$ and the $s$-dimensional Hausdorff measure by $\mathcal{H}^{s}$. The Hausdorff dimension of a set $X$ will be denoted by $\operatorname{dim}_{\mathcal{H}}(X)$, while the topological (Menger) dimension of $X$ will be denoted by $\operatorname{dim}_{\text {top }}(X)$. For more on these notions, see [20] and [18, Chapter 7]. A property holds for almost all points if the set of points for which the property does not hold has measure zero. If we do not state the measure which we are using, the phrase "almost all" will be construed to mean almost all with respect to the relevant Lebesgue measure.

The Lebesgue lower density of the set $E \subset \mathbb{R}^{n}$ at the point $x$ is the 
quantity

$$
\Phi(E, x)=\liminf _{r \rightarrow 0} \frac{\lambda_{n}(E \cap B(x, r))}{\lambda_{n}(B(x, r))} .
$$

A theorem of Lebesgue (see [35]) states that for every measuarable set $E$, this quantity is 1 at almost all points of $E$ and 0 at almost all points outside of $E$. A set is density open if its Lebesgue lower density is 1 at all of its points. The family of all density open sets is called the density topology. A deep theorem about the density topology is that this topology is regular. Temporarily denote the density topology on $\mathbb{R}^{n}$ by $T_{D}$ and the natural topology on $\mathbb{R}^{n}$ by $T$. A function $f: \mathbb{R}^{n} \rightarrow \mathbb{R}^{n}$ is called approimxately continuous if the function $f:\left(\mathbb{R}^{n}, T_{D}\right) \rightarrow\left(\mathbb{R}^{n}, T\right)$ is continuous. More on the density topology and approximately continuous functions can be found in [13] and [5].

We use the following classical notions to describe the continuity of functions. The oscillation of a function $f$ on a set $E$ is defined as

$$
\operatorname{osc}(f, E)=\sup _{x \in E} f(x)-\inf _{x \in E} f(x)
$$

and the oscillation of a function $f$ at a point $x$ is defined by

$$
\operatorname{osc}(f, x)=\lim _{\delta \rightarrow 0^{+}} \operatorname{osc}\left(f, B_{\delta}(x)\right)
$$

Note that $f$ is continuous at $x$ if and only if $\operatorname{osc}(f, x)=0$. Details may be found in [35, Chap. 7]. We also make use of the modulus of continuity

$$
\omega(f, \delta)=\sup \{|f(x)-f(y)|:\|x-y\|<\delta\}
$$

A function is uniformly continuous if and only if $\lim _{\delta \rightarrow 0^{+}} \omega(f, \delta)=0$. For 
details, see [4].

An isometry is a mapping, $\phi$, between metric spaces, $\left(X, d_{1}\right)$ and $\left(Y, d_{2}\right)$, so that for all $x_{1}, x_{2}, \in X, d_{1}\left(x_{1}, x_{2}\right)=d_{2}\left(\phi\left(x_{1}\right), \phi\left(x_{2}\right)\right)$. We say that two subsets of $\mathbb{R}^{n}$ are isometric provided there is an isometry of $\mathbb{R}^{n}$ whichs maps one onto the other. We note that a $k$-flat is a set in $\mathbb{R}^{n}$ isometric to $\mathbb{R}^{k}$. We denote the class of all $k$-flats in $\mathbb{R}^{n}$ by $\mathcal{F}_{k}\left(\mathbb{R}^{n}\right)$. Thus the class of all lines in $\mathbb{R}^{n}$ is written as $\mathcal{F}_{1}\left(\mathbb{R}^{n}\right)$ and the class of all hyperplanes in $\mathbb{R}^{n}$ is denoted $\mathcal{F}_{n-1}\left(\mathbb{R}^{n}\right)$. The class of all $k$-flats in $\mathbb{R}^{n}$ which are parallel to the flat spanned by some collection of the coordinate axes will be written $\mathcal{F}_{k}^{+}\left(\mathbb{R}^{n}\right)$. Again, if the underlying space is obvious, we suppress its mention. When not in the context of flats, the class of lines in $\mathbb{R}^{n}$ is sometimes denoted by $\mathcal{L}\left(\mathbb{R}^{n}\right)$ or as just $\mathcal{L}$ if the underlying space is understood. We note that a set of at least $k+1$ points of $\mathbb{R}^{n}$ is in general position if no $k-1$-flat contains $k$ of them. A set is convex if for each pair of points $x, y \in X$, the line segment $\overline{x y}$ is contained entirely in $X$. Then convex hull of a set $S, \operatorname{conv}(S)$ is the smallest convex set containing $S$. For more on these notions, see any text on convexity, for instance [22] or [28].

\subsection{History}

Despite the fact that there are many interesting results in the area of restriction continuities, it is worth mentioning that 200 years ago, the idea that such functions might exist was not even entertained. In his Cours d'Analyse [6], Cauchy stated that a function $f: \mathbb{R}^{2} \rightarrow \mathbb{R}$ is continuous if and only if it is continuous in each variable separately. The first known counterexample 
to this statement was published by J. Thomae (though given by Heine, see [38]) in an 1870 treatise on complex analysis [46]. The function in Thomae's text,

$$
f(x, y)= \begin{cases}\sin \left(4 \tan ^{-1}\left(\frac{x}{y}\right)\right) & \text { if } y \neq 0 \\ 0 & \text { if } y=0\end{cases}
$$

is continuous in each variable, since for any fixed $x, \lim _{y \rightarrow 0} 4 \tan ^{-1}\left(\frac{x}{y}\right)=\pi$. However, this function is discontinuous at the origin, since every nieghborhood of the origin contains points of the diagonal $(x, x)$, and at these points $f$ takes on the value $f(x, x)=\sin \left(4 \tan ^{-1}(1)\right) \neq 0$. During the later half of the nineteenth century, as the field which we would now call real analysis was being born, several types of what we have called restriction continuities arose in research. In particular, in 1884, a calculus treatise [21] by A. Genocchi and G. Peano (however, primarily due to Peano, see [38]) first noted the existance of discontinuous, but linearly continuous functions, giving as an example the function from equation (1.2).

As analysis matured, studies on restriction continuities began to focus on several different questions. One strand of thought, primarily advanced by Baire and Lebesgue, was focused on the regularity of $\mathcal{S}$-continuous functions. Another. spearheaded by Scheefer and Lebesgue began studying how large a class $\mathcal{S}$ could be while still allowing for a discontinuous $\mathcal{S}$-continuous functions. The last branch, perhaps most represented by the work of H. Hahn and the husband and wife team, W.H. and G.C. Young, consisted of finding how large a set of discontinuities that $\mathcal{S}$-continuous functions could have for various $\mathcal{S}$. In this work, we mostly concern ourselves with the first and third 
branches. The reader interested in the second is referred to [16] for a modern paper pursuing this topic.

The study of regularity of $\mathcal{S}$-continuous functions saw its first major result, according to Baire [2], when V. Volterra noticed that every separately continuous function $f: \mathbb{R}^{2} \rightarrow \mathbb{R}$ satisfied a topological condition called quasicontinuity. A function $f: X \rightarrow Y$ is quasi-continuous at a point $x$ if for every pairs of open sets $U \subset X$ and $W \subset Y$, with $x \in U$ and $f(x) \in W$, there is a nonempty open subset $V \subset U$ such that $f[V] \subset W$. Note that this differs from continuity only in the fact that the point $x$ need not be in $V$. A function is quasi-continuous if and only if it is quasi-continuous at every point. Baire furthered this train of thought, showing that every separately continuous function $f: \mathbb{R}^{2} \rightarrow \mathbb{R}$ is of the first Baire class, and that there is a residual set of lines parallel to each axis and such that each point of these lines is a continuity point of $f$. Baire's results were generalized by H. Lebesgue, who after independently discovering Baire's result on the Baire category of separately continuous functions defined on $\mathbb{R}^{2}[29]$, continued the proof inductively [30] to show that if $f: \mathbb{R}^{n} \rightarrow \mathbb{R}$ is separately continuous, then $f \in \mathcal{B}_{n-1}$. In this paper, he also proved that this was sharp; in particular, we have the following theorem.

Theorem 1.3.1 If $f: \mathbb{R}^{n} \rightarrow \mathbb{R}$ is separately continuous, then $f \in \mathcal{B}_{n-1}$. Conversely if $f: \mathbb{R} \rightarrow \mathbb{R}$, and $f \in \mathcal{B}_{n}$, then there exists a separately continuous $F: \mathbb{R}^{n+1} \rightarrow \mathbb{R}$ with $F(x, x, \ldots, x)=f(x)$. Hence the set $\mathcal{B}_{n}(\mathbb{R})$ is equal to the set of all functions obtained by restricting separately continuous functions on $\mathbb{R}^{n+1}$ to the diagonal.

The first half of this statement and several generalizations of it, may easily 
be inferred from the following easy lemma, first brought to the attention of the author in this general form by Prof. Z. Piotrowski (see [36]).

Lemma 1.3.2 Let $X, Y$, and $Z$ be metric spaces. If $f: X \times Y \rightarrow Z$ given by $\langle x, y\rangle \mapsto f(x, y)$ is such that

- $f(\cdot, y) \in \mathcal{C}$ for all $y_{0} \in Y$ and

- $f(x, \cdot) \in \mathcal{B}_{\alpha}$ for every $x \in X$

then $f \in \mathcal{B}_{\alpha+1}$.

The reader interested in spaces of infinite dimension should know that Lebesgue's result fails spectacularly in this context. It has been shown by Marczewski and Ryll-Nardzewski [31] that given an arbitrary function $f: \mathbb{R} \rightarrow \mathbb{R}$, there is a separately continuous function $F: \mathbb{R}^{\infty} \rightarrow \mathbb{R}$ such that $F(x, x, \ldots)=f(x)$.

It worth noting that no concerted effort appears to have been made to discuss the Baire class of functions satisfying restriction continuity conditions other than that of separate continuity. This is most likely because for most classes $\mathcal{S}$ of interest in this study, the class $\mathcal{C}_{\mathcal{S}}$ is contained in the class of separately continuous functions. Note that it follows that any separately (and hence linearly, etc.) continuous function is measurable.

The second stream of thought began taking shape around the work of Scheefer [43] and Lebesgue [30] who showed (in two different contexts) that a function could be continuous along every analytic path yet be discontinuous, i.e., that the class of $\mathfrak{C}(\mathcal{A})$-continuous functions does not coincide with the 
class of continuous functions. The natural question to ask at this point was "For which classes of graphs $F$, does $F$-continuity imply continuity?"

Progress on this question came from Lusin, who proved that $\mathfrak{C}(C)$-continuity is the same continuity. In particular, he proved the following result. For more information, and generalizations, see [17].

Theorem 1.3.3 Let $\mathcal{F}$ be the collection of continuous functions $[a, b] \rightarrow$ $[c, d]$ and continuous functions $[c, d] \rightarrow[a, b]$. Then any function $f:[a, b] \times$ $[c, d] \rightarrow \mathbb{R}$ is continuous if and only if it is F-continuous.

The question of which families, $F$, of graphs have the property that $F$ continuity coincides with continuity was almost fully answered by Rosenthal in 1955 (see [41]).

Theorem 1.3.4 If $f: \mathbb{R}^{n} \rightarrow \mathbb{R}$ is $\mathfrak{C}\left(C^{1}\right)$-continuous, then $f$ is continuous, however, there is a discontinuous function, which is $\mathfrak{C}\left(\mathcal{C}^{2}\right)$-continuous.

In fact, Rosenthal proves more; if one only checks that every restriction of $f: \mathbb{R}^{2} \rightarrow \mathbb{R}$ to some convex $C^{1}$ curve, then $f$ is continuous. In higher dimensions, the same is true with "convex" replaced by a condition Rosenthal names "primitive", which is a recursively defined condition on the projections of a curve, which reduces to convexity in the case the curve is a subset of $\mathbb{R}^{2}$.

The importance of our definition of curve in the above theorem is made salient by contrasting the theorem to the following special case of a theorem of Boman (see [3] or [24] for details).

Theorem 1.3.5 Let $F: \mathbb{R}^{2} \rightarrow \mathbb{R}$ be a function and assume that for all $\mathcal{C}^{\infty}$ functions $h_{1}, h_{2}: \mathbb{R} \rightarrow \mathbb{R}$ the composition $F \circ\left\langle h_{1}, h_{2}\right\rangle$ is continuously differentiable. Then $F$ is continuous. 
Boman's result does not contradict Rosenthal's since according to the definition of $\mathcal{C}^{1}$ used in Rosenthal's results, our curves must have a nonvanishing tangent at every point, while Boman's curves need only be infinitely differentiable. In the language of differential geometry, Rosenthal's curves are immersions of the interval, while Boman's are merely infinitely differentiable images of the interval.

The third line of research in the area of restriction continuities concerns the structure of their discontinuity sets. It appears that the first step toward understanding these sets came from Baire [2], who in 1899 showed that there is a separately continuous function $f: \mathbb{R}^{3} \rightarrow \mathbb{R}$ such that $D(f)$ contains a line segment. This result was expanded by Hahn [23] who observed that if $f: \mathbb{R}^{2} \rightarrow \mathbb{R}$ is the function from expression (1.1), then the function $g: \mathbb{R}^{n} \rightarrow$ $\mathbb{R}$ defined by

$$
g\left(x_{1}, x_{2}, \ldots x_{n}\right)=f\left(x_{1}, x_{2}\right)
$$

is discontinuous at every point of the $(n-2)$-dimensional region $x_{1}=x_{2}=0$; hence showing that $D(f)$ may contain $(n-2)$-dimensional rectangles for separately continuous $f$.

The problem of deciding "how discontinuous" a separately continuous function can be was settled by Kershner [25], who, in 1943, characterized the sets of discontinuities of separately continuous functions with the following theorem.

Theorem 1.3.6 For any set $D \in \mathbb{R}^{n}$ there is a separately continuous function $f: \mathbb{R}^{n} \rightarrow \mathbb{R}$ with $D(f)=D$ if, and only if, $D$ is an $F_{\sigma}$ set and every orthogonal projection of $D$ onto a coordinate hyperplane is of first category. 
Note that the discontinuity set of any function is an $F_{\sigma}$ set (or see [35]), hence the only new condition imposed by this theorem is that the projections be small. Many generalizations of this theorem exist, including versions valid on various topological spaces. The reader will find [4] and the historical survey therein particularly illustrative of the nature of these results.

Studies on the structure of $D(f)$ where $f$ is linearly continuous exist, but they are not as penetrating as those for separately continous functions. One of the earliest such results can be found in a paper by W.H. and G.C. Young $([47])$, published at the dawn of the twentieth century which exhibited many linearly continuous functions with large discontinuity sets. The most impressive example in this paper is a function $f:[0,1] \times[0,1] \rightarrow \mathbb{R}$ for which $D(f)$ is uncountable and dense in every open subset of its domain. Linearly continuous functions were little studied after Young and Young's paper, until 1944, when A. S. Kronrod took a course on the theory of real functions from N. Lusin (by then a rare opportunity.) The course influenced him to begin a research program toward the development of a geometric theory of real functions (see [27]). This research program led to Kronrod asking for a description of the set of discontinuities of linearly continuous functions defined on the plane. The first partial answer to Kronrod's challenge came from S. Slobodnik [45], who gave the following necessary condition for a set $D$ to be a discontinuity set of some linearly continuous function.

Theorem 1.3.7 If $D=D(f)$ for some linearly continuous function $f: \mathbb{R}^{n} \rightarrow$ $\mathbb{R}$, then $D=\bigcup_{i=1}^{\infty} D_{i}$, where each $D_{i}$ is isometric to the graph of a Lipshitz function defined on a nowhere dense subset of $\mathbb{R}^{n-1}$.

Being as the $n$-dimensional Lebesgue measure of the graph of a Lipshitz 
function defined on an $(n-1)$-dimensional space is 0 (see [20]), Slobodnik's result tells us that if $f$ is linearly continuous, then $\lambda_{n}(D(f))=0$ (recall $\lambda_{n}$ is $n$-dimensional Lebesgue measure), and that $D(f)$ is of the first category. Hence, although separately continuous functions can have discontinuity sets of full measure (see [45]), the discontinuity set of a linearly continuous function cannot. Furthermore, Slobodnik proved that various projections of the sets $D \in \mathcal{D}_{1, n}$ are "small".

Theorem 1.3.8 Let $f: \mathbb{R}^{n} \rightarrow \mathbb{R}$ be linearly continuous. In the decomposition in Theorem 1.3.7 of $D(f)$, the orthogonal projection of any $D_{i}$ onto any hyperplane is nowhere dense in this hyperplane. Furthermore, for every $x \in D_{i}$ is the central projection of $\mathcal{D}_{i} \backslash\{x\}$ through $x$ onto any hyperplane in $\mathbb{R}^{n} \backslash\{x\}$ is nowhere dense in that hyperplane.

The central projection through $x$ onto a hyperplane of the above theorem is defined as follows, for a point $x \in \mathbb{R}^{n}$ and hyperplane $H \subset \mathbb{R}^{n} \backslash\{a\}$, the central projection is the set $C_{a} H \cap H$ where $C_{a} H$ is the set of all rays $\overrightarrow{a b}$, with $b \in H$. Slododnik's response to Kronrod's challenge would be the only voice in this direction for twenty years. However, in 1997, E. E. Shnol', recalling some conversations with Kronrod and evidently unaware of Slobodnik's results (or, for that matter, Kershner's), proved a necessary condition very much like Slobonik's Theorem 1.3.8 (incorrectly listed as necessary and sufficient in the English translation) on the sets of $\mathcal{D}_{1,2}$ [44].

Theorem 1.3.9 A set $F \subset \mathbb{R}^{2}$ is a set of the form $\{\vec{x}=\langle x, y\rangle: \operatorname{osc}(f, \vec{x}) \geq a\}$ only if the set of directions in which points of $F$ are seen from any point $O$ constitutes a nowhere dense set. 
In the above theorem, a point $P \in F$ can be seen from a point $Q$ in a direction $\alpha$ if there is a line segment from $Q$ to $P$ in the direction $\alpha$. Note that this is basically the central projection of Theorem 1.3.8, except that instead of projecting onto a hyperplane, we are projecting onto an $(n-1)$-sphere. The result of Shnol' can be found in more general form in Slobodnik's work, however, Shnol's paper gives much attention to the construction of many sets fitting his description, which is absent in Slobodnik. Shnol', following Kronrod's interests, paid no attention to this problem in dimension higher than 2 , and in fact, his methods make heavy use of the fact that in $\mathbb{R}^{2}$, the dimension and codimension of a line both equal 1 , and hence these methods cannot be directly extended to higher dimensional cases. 


\section{Chapter 2}

\section{Basic Results}

\section{$2.1 \quad$ Introduction}

In this chapter, we gather some basic results for restriction continuities, in some generality. The results are not difficult; however, they do originate with this document. While we note that, throughout the literature, the study of restriction continuities has focused on geometrically natural subsets of $\mathbb{R}^{n}$ or their obvious generalization to more general spaces, in order to add breadth and perspective to our study, we strive for maximum generality in this section. To keep our discussion as broad as possible, we work with topological spaces $X$ and $Y$, imposing as few conditions imposed on them as necessary. We will also assume the family $\mathcal{S}$ of subsets of $X$ to be nonempty so as to not have to tiresomely repeat a consideration of a trivial case. The first thing which we wish to note is that all restriction continuities are generalizations of continuity. 
Proposition 2.1.1 Let $X$ and $Y$ be topological spaces. Let $\mathcal{S}$ be a family of subsets of $X$. If $f: X \rightarrow Y$ is continuous, then $f$ is $\mathcal{S}$-continuous.

Although, we have a definition of restriction continuity, note that we could give the following as an alternative definition.

Definition 2.1.2 Let $X$ and $Y$ be topological spaces. Let $\mathcal{S}$ be a family of subsets of $X$. A function $f: X \rightarrow Y$ is $\mathcal{S}$-continuous at a point $p \in X$ if for every open set $V \subset Y$ containing $f(p)$, there is an open set $U \subset X$ such that $p \in U, f[U \cap S] \subset V$ for every $S \in \mathcal{S}$ containing $p$.

When convenient, we will use this definition.

Proposition 2.1.3 Let $X$ and $Y$ be topological spaces. Let $\mathcal{S}$ be a family of subsets of $X$. If $f: X \rightarrow Y$ is continuous at a point $x$, then $f$ is $\mathcal{S}$-continuous at $x$.

Propositions 2.1.1 and 2.1.3 follow immediately from the definitions of continuity and continuity at a point. Our next examples show that there are plenty of cases in which restriction continuities do not expand the notion of continuity at all.

Example 2.1.4 If $X$ and $Y$ are topological spaces and $\mathcal{S}=\{X\}$, then $\mathcal{S}$ continuity coincides with continuity. This also holds if $\mathcal{S}$ is any subbasis for the topology on $X$.

Example 2.1.5 If $X$ is a first-countable topological space, $Y$ is any topological space, and $\mathcal{S}$ is the set of all convergent sequences of points of $X$ together with their limits, then $\mathcal{S}$-continuity and continuity coincide. This 
follows from the fact, see $[18$, p. 78$]$ that sequences are sufficient to dectect the continuity points of a function defined on a first-countable space.

Conversely, the following example shows we can find $\mathcal{S}$ such that $\mathcal{S}$ continuity is more of a generalization than is useful.

Example 2.1.6 If $X$ and $Y$ are topological spaces and $\mathcal{S}$ is the family of all singleton subsets of $X$, then every function $f: X \rightarrow Y$ is $\mathcal{S}$-continuous. The same is true is true if every element of $\mathcal{S}$ is a set all of whose points are isolated.

The three above examples show that restriction continuity is not always an interesting notion at all. The study of restriction continuities will have to focus on those choices of $\mathcal{S}$ which fall between the extreme of not generalizing continuity at all and generalizing it too far, including all possible functions.

\subsection{Basic Theorems}

The primary focus of this section will be on the relation between the family $\mathcal{S}$ and the set of $\mathcal{S}$-continuous functions. The first difficulty in this study comes in the following form. If $\mathcal{P}$ represents the power set operation and $F(X, Y)$ represents the set of all functions from $X$ to $Y$, then we may consider the mapping $\Psi: \mathcal{P}(\mathcal{P}(X)) \rightarrow \mathcal{P}(F(X, Y))$, defined by $\Psi(\mathcal{S})=\mathcal{C}_{\mathcal{S}}$, where we use our convention $\mathcal{C}_{\mathcal{S}}=\{f: X \rightarrow Y: f$ is $\mathcal{S}$-continuous $\}$. This function is not injective, since given any collection $\mathcal{S}$, we may add any number of singleton sets to it without changing $\mathcal{C}_{\mathcal{S}}$. To explore this facet of the theory, we prove a theorem which explains, at least to some extent, how "non-injective" the 
mapping $\Phi$ is. The first of which we have already informally stated and which follows easily from Example 2.1.6.

Lemma 2.2.1 Let $X$ and $Y$ be topological spaces and let $\mathcal{S}$ be a family of subsets of $X$. Let $\mathcal{S}_{0}$ be the union of the family $\mathcal{S}$ with the family of all singleton subset of $X$. Then the classes $\mathcal{C}_{\mathcal{S}}$ and $\mathcal{C}_{\mathcal{S}_{0}}$ coincide.

We state several simple related lemmas to prove a theorem which will simplify our approach.

Lemma 2.2.2 If $f: X \rightarrow Y$ is a function such that $f\lceil S$ is continuous for some $S \subset X$ and $T$ is any nonempty subset of $S$, then $f\lceil T$ is continuous. Furthermore, if $f\lceil T$ is continuous for every subset of $T$ of $S$, then $f\lceil S$ is continuous.

Proof. One direction follows trivially from the fact that inclusion maps are continuous and compositions of continuous functions are continuous and the other is a consequence of the fact that for any set $S$, we have $S \subset S$.

Lemma 2.2.3 If $f: X \rightarrow Y$ is a function s such that there exist closed sets $M$ and $N$, with $f\lceil M$ and $f \uparrow N$ continuous, then $f \uparrow(M \cup N)$ is continuous.

Proof. Let $C \subset Y$ be closed. Then $C_{1}=f^{-1}(C) \cap M$ and $C_{2}=f^{-1}(C) \cap N$ are closed. Hence $C_{1} \cup C_{2}=f^{-1}(C) \cap(M \cup N)$ is closed.

Lemma 2.2.4 If $f: X \rightarrow Y$ is a function between topological spaces such that there exists a collection $\left\{U_{\alpha}: \alpha \in \mathcal{A}\right\}$ of open subsets of $X$ where $f \nmid U_{\alpha}$ is continuous for each $\alpha \in \mathcal{A}$, then $f \uparrow\left(\bigcup_{\alpha \in \mathcal{A}} U_{\alpha}\right)$ is continuous. 
Proof. Let $V \subset Y$ be open. Then $V_{\alpha}=f^{-1}(V) \cap U_{\alpha}$ is open for each $\alpha \in \mathcal{A}$. Then $\bigcup_{\alpha \in \mathcal{A}} V_{\alpha}$ is open in $\bigcup_{\alpha \in \mathcal{A}} U_{\alpha}$.

Combining these lemmas, we arrive at the following theorem.

Theorem 2.2.5 Let $X$ and $Y$ be topolgical spaces. Let $\mathcal{S}$ be a family of subsets of $X$. Let $\mathcal{S}^{*}$ be the family generated from $X$ by adding to it all singleton subsets of $X$, the union of any finite number of closed subsets of $\mathcal{S}$ and the union of any collection of open elements of $\mathcal{S}$, and then closing this collection under taking subsets. Then $\mathcal{S}$-continuity and $\mathcal{S}^{*}$-continuity coincide.

We may therefore always assume that $\bigcup \mathcal{S}=X$ and if convenient, we may choose to only study the families $\mathcal{S}^{*}$. Furthermore, if we consider any family of subsets $\mathcal{T}$ with $\mathcal{S} \subset \mathcal{T} \subset \mathcal{S}^{*}$, then $\mathcal{S}$-continuity and $\mathcal{T}$-continuity coincide.

Note that we only include unions of open and closed sets. That we cannot include unions of arbitrary sets is a consequence of the following example.

Example 2.2.6 Let $X=Y=\mathbb{R}$. Let $\mathcal{S}=\{\mathbb{Q}, \mathbb{R} \backslash \mathbb{Q}\}$. Then the characteristic function of the rationals, $\chi_{\mathbb{Q}}$ is $\mathcal{S}$-continuous, however, if we extend $\mathcal{S}$ to a family $\mathcal{T}$ which includes the union of $\mathbb{Q}$ and $\mathbb{R} \backslash \mathbb{Q}$, i.e. $\mathbb{R}$, then $\chi_{\mathbb{Q}}$ is not $\mathcal{T}$-continuous.

Having made the reduction of Theorem 2.2.5, we will now focus on the problem of combining families of sets in various ways and which functions are continuous when restricted to them. Let $\mathcal{S}_{1}$ and $\mathcal{S}_{2}$ be two families of subsets of $X$.

The following proposition is an obvious consequence of Theorem 2.2.5. 
Proposition 2.2.7 If $\mathcal{S}_{1}$ and $\mathcal{S}_{2}$ are collections of subsets of $X$ such that for every $S \in \mathcal{S}_{1}$, there is a $T \in \mathcal{S}_{2}$ such that $S \subset T$. Then $\mathcal{C}_{\mathcal{S}_{2}} \subset \mathcal{C}_{\mathcal{S}_{1}}$.

Proposition 2.2.8 $\mathcal{C}_{\mathcal{S}_{1} \cup \mathcal{S}_{2}}=\mathcal{C}_{\mathcal{S}_{1}} \cap \mathcal{C}_{\mathcal{S}_{2}}$

PRoOF. If $f \in \mathcal{C}_{\mathcal{S}_{1}} \cap \mathcal{C}_{\mathcal{S}_{2}}$, then $f\left\lceil S\right.$ is continuous for any $S \in \mathcal{S}_{1} \cup \mathcal{S}_{2}$, hence $f \in \mathcal{C}_{\mathcal{S}_{1} \cup \mathcal{S}_{2}}$. The opposite inclusion is proved by noting that if $f\lceil S$ is continuous for any $S \in \mathcal{S}_{1} \cup \mathcal{S}_{2}$, then $f \in \mathcal{C}_{\mathcal{S}_{i}}$ for $i=1,2$.

Similarly proved is the following proposition.

Proposition 2.2.9 Let $\mathcal{S}_{1}$ and $\mathcal{S}_{2}$ be families of subsets of a set $X$. Then $\mathcal{C}_{\mathcal{S}_{1}} \cup \mathcal{C}_{\mathcal{S}_{2}} \subset \mathcal{C}_{\mathcal{S}_{1} \cap \mathcal{S}_{2}}$

However, equality need not hold, as shown by the following example.

Example 2.2.10 Consider, for simplicity $X=\mathbb{R}$. Let $\mathcal{S}_{1}=\{\mathbb{Q}, \mathbb{R}\}$, while letting $\mathcal{S}_{2}$ be the set of all (Lebesgue) measure zero subsets of $\mathbb{R}$. Then the characteristic function of the rationals is in $\mathcal{C}_{\mathcal{S}_{1} \cap \mathcal{S}_{2}}$, however, it is clearly not in $\mathcal{C}_{\mathcal{S}_{1}}$. It is not in $\mathcal{C}_{\mathcal{S}_{2}}$, since the set $\mathbb{Q} \cup\{\pi\}$ is of measure zero.

Before we close this section, we note the basic relationships that hold between $\mathcal{S}, \mathcal{C}_{\mathcal{S}}$, and $\mathcal{D}_{\mathcal{S}}$.

Proposition 2.2.11 $\mathcal{S}_{1} \subset \mathcal{S}_{2} \Longrightarrow \mathcal{C}_{\mathcal{S}_{2}} \subset \mathcal{C}_{\mathcal{S}_{1}}$.

Proof. Let $\mathcal{S}_{1}$ and $\mathcal{S}_{2}$ be two collections of subsets of $X$, with $\mathcal{S}_{1} \subset \mathcal{S}_{2}$. Let $f: X \rightarrow Y$ be $\mathcal{S}_{2}$ continuous. Since every $S \in \mathcal{S}_{1}$ is also an element of $\mathcal{S}_{2}$, $f \uparrow S$ is continuous for every $S \in \mathcal{S}_{1}$. Hence $f$ is $\mathcal{S}_{1}$-continuous. 
Similarly, assume that there are two collections of subsets of $X, \mathcal{S}_{1}$ and $\mathcal{S}_{2}$ are such that $\mathcal{C}_{\mathcal{S}_{1}} \subset \mathcal{C}_{\mathcal{S}_{2}}$. Then since every function $f$ in $\mathcal{C}_{\mathcal{S}_{1}}$ is in $\mathcal{C}_{\mathcal{S}_{2}}$, and so $D(f) \in \mathcal{D}_{\mathcal{S}_{2}}$. Hence the following result.

Proposition 2.2.12 $\mathcal{C}_{\mathcal{S}_{1}} \subset \mathcal{C}_{\mathcal{S}_{2}} \Longrightarrow \mathcal{D}_{\mathcal{S}_{1}} \subset \mathcal{D}_{\mathcal{S}_{2}}$.

We note, before leaving this section, one final result, related to our first topic in this section. We noted the mapping $\Psi: \mathcal{P}(\mathcal{P}(X)) \rightarrow \mathcal{P}(F(X, Y))$ defined by $\Psi(\mathcal{S})=\mathcal{C}_{\mathcal{S}}$ is not, in general, a bijection. We also note that the partial mapping $\Phi: \mathcal{P}(F(X, \mathbb{R})) \rightarrow \mathcal{P}(\mathcal{P}(X))$ defined by $\Phi\left(\mathcal{C}_{\mathcal{S}}\right)=\mathcal{D}_{\mathcal{S}}$ is not, in general, a bijection. For instance, let $X=\mathbb{R}^{2}$ and consider the set $\mathcal{S}_{1}$ consist of all lines parallel to either the $x$ or $y$ axis not passing through the origin, as well as both the $x$ and $y$ axes with their origins deleted. Let $\mathcal{S}_{2}$ consist of all lines parallel to either the $x$ or $y$ axis.

Although functions such as

$$
f(x, y)= \begin{cases}\frac{2 x y}{x^{2}+y^{2}} & \text { if }\langle x, y\rangle \neq\langle 0,0\rangle \\ 1 & \text { if }\langle x, y\rangle=\langle 0,0\rangle .\end{cases}
$$

exist which are in $\mathcal{C}_{\mathcal{S}_{1}}$ but not $\mathcal{C}_{\mathcal{S}_{2}}$, every such function is identical to some separately continuous function at all points except the origin. So if $f \in$ $\mathcal{C}_{\mathcal{S}_{1}} \backslash \mathcal{C}_{\mathcal{S}_{2}}$ and if $g \uparrow\left(\mathbb{R}^{2} \backslash\langle 0,0\rangle\right)=f \uparrow\left(\mathbb{R}^{2} \backslash\langle 0,0\rangle\right)$ then $D(f) \backslash D(g)$ consists of at most one point. However, $D(g)$ is an $F_{\sigma}$ set whose projections are of the first category. Since the singleton consisting of just the origin is closed $D(g) \cup$ $\{\langle 0,0\rangle\}$ is also an $F_{\sigma}$ set. Since the projections of $D(g)$ are first category, their union with the nowhere dense set $\{\langle 0,0\rangle\}$ remains first category. So $D(f)=D(g) \cup\{\langle 0,0\rangle\}$ is an $F_{\sigma}$ set whose projections are first category, and 
hence is the set of discontinuities of some separately continuous function. Thus $\Phi$ is not bijective.

\section{$2.3 \quad$ A Basic Lemma}

In this section, we present a lemma which will enable us to construct many discontinuous functions which obey some form of restriction continuity. In almost every subsequent chapter, we will use some form of this lemma, usually, with $\mathbb{R}^{n}$ standing in for $X$ and with some family of curves or surfaces standing in for $\mathcal{S}$.

Lemma 2.3.1 Let $\mathcal{S}$ be a family of sets in some metric space $X$ and let $\left\{D_{j} \subset X: j<\omega\right\}$ be a pointwise finite family (that is, each point is contained in only finitely many of the elements of the family) of open sets in $X$ such that

(F) the set $\left\{j<\omega: D_{j} \cap S \neq \emptyset\right\}$ is finite for every $S \in \mathcal{S}$.

Then for every sequence $\left\langle F_{j}: j<\omega\right\rangle$ of continuous functions from $X$ into $\mathbb{R}$ such that $\operatorname{supp}\left(F_{i}\right) \subset D_{i}$ for all $i<\omega$, the function $F \stackrel{\text { def }}{=} \sum_{j<\omega} F_{j}$ is $\mathcal{S}$-continuous. Moreover, if

- the $D_{j}$ 's are pairwise disjoint

- the diameters of the sets $D_{j}$ go to 0 , as $j \rightarrow \infty$,

- $\hat{P}$ is the set of all $z \in X$ for which every open $U \ni z$ intersects infinitely many sets $D_{j}$, and

- each function $F_{j}$ is onto $[0,1]$, 
then $\hat{P}=D(F)=\left\{z \in X: \omega_{F}(z)=1\right\}$.

Proof.The first part is obvious. The second follows easily from the fact, that, for any $z \in \hat{P}$, every open $U \ni z$ contains infinitely many sets $D_{j}$. 


\section{Chapter 3}

\section{$\mathfrak{C}\left(\mathcal{D}^{2}\right)$-continuous Functions with Large Discontinuity Sets}

\subsection{Introduction}

This chapter studies the $\mathfrak{C}\left(\mathcal{D}^{2}\right)$-continuous functions from $\mathbb{R}^{2} \rightarrow \mathbb{R}$, following closely the paper, [9]. Recall that the class $\mathfrak{C}\left(\mathcal{D}^{2}\right)$ is the class of all $\mathcal{D}^{2}$ curves in the plane. These functions are important in light of Rosenthal's theorem that any function all of whose restrictions to $C^{1}$ curves are continuous is, itself a continuous function. Thus, $\mathfrak{C}\left(\mathcal{D}^{2}\right)$ is the largest differentiability class of curves which can "miss" a discontinuity. While Rosenthal produced an example of a $\mathfrak{C}\left(\mathcal{C}^{2}\right)$-continuous function with a single point of discontinuity, he neither indicated the possible size of $D(f)$ for $\mathfrak{C}\left(\mathcal{C}^{2}\right)$-continous $f$, nor $\operatorname{did}$ he discuss the whether or not there exist $\mathfrak{C}\left(\mathcal{D}^{2}\right)$ continuous functions. The goal of this chapter is to clarify these points. We note that since any line in $\mathbb{R}^{n}$ is a $\mathcal{C}^{2}$ curve, any $\mathfrak{C}\left(\mathcal{C}^{2}\right)$-continuous function is linearly continuous. Hence 
Slobodnik's theorem, Theorem 1.3.7 tells us that if $f$ is $\mathfrak{C}\left(\mathcal{C}^{2}\right)$-continuous on $\mathbb{R}^{n}$, then the Hausdorff dimension of $D(f)$ cannot exceed $n-1$. In particular, in $\mathbb{R}^{2}$, the Hausdorff dimension of $D(f)$ for such an $f$ is no more than 1 .

Below, we construct a $\mathfrak{C}\left(\mathcal{D}^{2}\right)$-continuous (and hence $\mathfrak{C}\left(\mathcal{C}^{2}\right)$-continuous) function on $\mathbb{R}^{2}$ whose discontinuity set is perfect. Note that in light of Kershner's theorem (Theorem 1.3.6), this is as large as it can be, from the point of view of topology; we cannot create a second category example of such a set. Furthermore, we are able to show that the 1-dimensional Hausdorff measure of $D(f)$ can be an arbitrarily large finite number. Hence, we answer both questions with one function, in that there do exist discontinuous $\mathfrak{C}\left(D^{2}\right)$ continuous functions and that the Slobodnik's upper bound on the Hausdorff dimension of $D(f)$ is sharp. The construction of our function requires some complicated induction procedures. For the sake of simplicity, we show that elementary methods (of the type explainable to an undergraduate) may be used to construct a $\mathfrak{C}\left(\mathcal{D}^{2}\right)$-continuous function with a perfect discontinuity set, however, the sets $D(f)$ from these more simply constructed functions must satistfy $\mathcal{H}^{1}(D(f))=0$. Hence they do not so readily demonstrate the sharpness of Slobodnik's upper bound.

\subsection{The First Construction}

We will construct in this section a $\mathfrak{C}\left(\mathcal{D}^{2}\right)$-continuous function $f: \mathbb{R}^{2} \rightarrow \mathbb{R}$ whose discontinuty set has positive but finite 1-dimensional Hausdorff measure. In order to construct the function, we will use Lemma 2.3 .1 with $\hat{P}=h \uparrow P$, the graph of $h$ restricted to $P$, where $h$ and $P$ are from the 
proposition below.

Lemma 3.2.1 For every $M \in[0,1)$ there exists a $\mathcal{C}^{1}$ function $h: \mathbb{R} \rightarrow \mathbb{R}$ and a nowhere dense perfect $P \subset(0,1)$ of measure $M$ such that for every $\hat{x} \in P:$

$$
h^{\prime}(\hat{x})=0 \text { and } \lim _{x \rightarrow \hat{x}} \frac{|h(x)-h(\hat{x})|}{(x-\hat{x})^{2}}=\infty .
$$

We will postpone the proof of Lemma 3.2.1 for now. However, we notice here, that the $\operatorname{limit}_{\lim } \rightarrow \hat{x} \frac{|h(x)-h(\hat{x})|}{(x-\hat{x})^{2}}$ is a variant of the $\operatorname{limit}_{\lim } \lim _{x \rightarrow \hat{x}} 2 \frac{h(x)-h(\hat{x})}{(x-\hat{x})^{2}}$, which constitutes a generalized second derivative (related to Peano derivative) of $h$ at $\hat{x}$. Indeed, if $h^{\prime \prime}(\hat{x})$ exists, finite or infinite, then, by l'Hôpital's Rule, $\lim _{x \rightarrow \hat{x}} 2 \frac{h(x)-h(\hat{x})}{(x-\hat{x})^{2}}=\lim _{x \rightarrow \hat{x}} 2 \frac{h^{\prime}(x)-0}{2(x-\hat{x})}=\lim _{x \rightarrow \hat{x}} \frac{h^{\prime}(x)-h^{\prime}(\hat{x})}{x-\hat{x}}=h^{\prime \prime}(\hat{x})$. We need Lemma 3.2.1 in its current form, since there is no $\mathcal{C}^{1}$ function $h$ having an infinite second derivative on a set of positive measure. ${ }^{1}$

The existance of the desired function is established in the following theorem, which follows from Lemma 3.2.1.

Theorem 3.2.2 Let $h$ and $P$ be as in Lemma 3.2.1. Then $\hat{P}=h \uparrow P$ is the set of points of discontinuity of a $\mathfrak{C}\left(\mathcal{D}^{2}\right)$-continuous function $F: \mathbb{R}^{2} \rightarrow \mathbb{R}$. Moreover, $F$ can be made to have oscillation equal to 1 at every point from $\hat{P}$.

ProOF. Let $\left\{J_{j}: j<\omega\right\}$ be an enumeration, without repetitions, of bounded connected components of $\mathbb{R} \backslash P$. For every $j<\omega$ let $I_{j}$ be the open middle third subinterval of $J_{j}$ and let $F_{j}$ be a continuous function from $\mathbb{R}^{2}$ onto $[0,1]$

\footnotetext{
${ }^{1}$ This follows, for example, from [1, thm. 19] (used with $f=h^{\prime}$ ) which says that: for any real-valued continuous function $f$ defined on a set $P \subset \mathbb{R}$ of positive measure there exists a $\mathcal{C}^{1}$ function $g: \mathbb{R} \rightarrow \mathbb{R}$ which agrees with $f$ on an uncountable set.
} 
with $\operatorname{supp}\left(F_{j}\right)$ contained in $D_{j}=\left\{\langle x, y\rangle \in \mathbb{R}^{2}: x \in I_{j} \&|y-h(x)|<\left|I_{j}\right|^{3}\right\}$, where $\left|I_{j}\right|$ is the length of $I_{j}$. We will show that the function $F=\sum_{j<\omega} F_{j}$ is as required.

It is enough to show that sets $D_{j}$ satisfy the property (F) from Lemma 2.3.1 for the set $\mathfrak{C}\left(D^{2}\right)$, since all other assumptions of Lemma 2.3.1 are clearly satisfied. This is made simpler in the case of $\mathbb{R}^{2}$ allowing $\mathcal{D}^{2}$ to be the set of all graphs of twice differentiable functions $y=f(x)$ and $\left(\mathcal{D}^{2}\right)^{-1}$ to be the set of the inverse relation of such graphs. Then, although it is not the case that $\mathfrak{C}\left(\mathcal{D}^{2}\right)=\mathcal{D}^{2} \cup\left(\mathcal{D}^{2}\right)^{-1}$ globally, this identity does hold in some neighborhood of any point on any $D^{2}$ curve, via the Implicit Function Theorem. This is enough for us to see that $\mathfrak{C}\left(\mathcal{D}^{2}\right)$-continuity and $\mathcal{D}^{2} \cup\left(\mathcal{D}^{2}\right)^{-1}$ continuity coincide.

To see that our sets $D_{j}$ satisfy property (F), fix a $D^{2}$ function $g: \mathbb{R} \rightarrow \mathbb{R}$. We need to prove that both $g$ and $g^{-1}$ intersect only finitely many sets $D_{j}$.

To see that $g$ intersects only finitely many sets $D_{j}$, by way of contradiction, assume that there is an infinite set $\left\{j_{n}: n<\omega\right\}$ such that $g \cap D_{j_{n}} \neq \emptyset$. For $n<\omega$ choose $\left\langle x_{n}, y_{n}\right\rangle \in g \cap D_{j_{n}}$. Then $g\left(x_{n}\right)=y_{n}$ for all $n<\omega$. Choosing a subsequence, if necessary, we can assume that $\lim _{n \rightarrow \infty} x_{n}=\hat{x} \in P$. Then, by the definition of sets $D_{j}$, we have

$$
\lim _{n \rightarrow \infty}\left(y_{n}-h\left(x_{n}\right)\right)=\lim _{n \rightarrow \infty} \frac{y_{n}-h\left(x_{n}\right)}{x_{n}-\hat{x}}=\lim _{n \rightarrow \infty} \frac{y_{n}-h\left(x_{n}\right)}{\left(x_{n}-\hat{x}\right)^{2}}=0,
$$

as $\lim _{n \rightarrow \infty}\left|\frac{y_{n}-h\left(x_{n}\right)}{\left(x_{n}-\hat{x}\right)^{2}}\right| \leq \lim _{n \rightarrow \infty} \frac{\left|y_{n}-h\left(x_{n}\right)\right|}{\left|I_{j_{n}}\right|^{2}} \leq \lim _{n \rightarrow \infty}\left|I_{j_{n}}\right|=0$. In particular,

$$
g(\hat{x})=\lim _{n \rightarrow \infty} g\left(x_{n}\right)=\lim _{n \rightarrow \infty} y_{n}=\lim _{n \rightarrow \infty}\left(y_{n}-h\left(x_{n}\right)\right)+\lim _{n \rightarrow \infty} h\left(x_{n}\right)=h(\hat{x})
$$


and

$$
g^{\prime}(\hat{x})=\lim _{n \rightarrow \infty} \frac{y_{n}-h(\hat{x})}{x_{n}-\hat{x}}=\lim _{n \rightarrow \infty} \frac{y_{n}-h\left(x_{n}\right)}{x_{n}-\hat{x}}+\lim _{n \rightarrow \infty} \frac{h\left(x_{n}\right)-h(\hat{x})}{x_{n}-\hat{x}}=h^{\prime}(\hat{x})=0 .
$$

Hence, by l'Hôpital's Rule, $\lim _{x \rightarrow \hat{x}} \frac{g(x)-g(\hat{x})}{(x-\hat{x})^{2}}=\lim _{x \rightarrow \hat{x}} \frac{g^{\prime}(x)-0}{2(x-\hat{x})}=\frac{1}{2} g^{\prime \prime}(\hat{x})$ and, using (3.2) once more,

$$
\lim _{n \rightarrow \infty} \frac{h\left(x_{n}\right)-h(\hat{x})}{\left(x_{n}-\hat{x}\right)^{2}}=\lim _{n \rightarrow \infty} \frac{h\left(x_{n}\right)-y_{n}}{\left(x_{n}-\hat{x}\right)^{2}}+\lim _{n \rightarrow \infty} \frac{g\left(x_{n}\right)-g(\hat{x})}{\left(x_{n}-\hat{x}\right)^{2}}=\frac{1}{2} g^{\prime \prime}(\hat{x}),
$$

where the first equation is justified by $y_{n}=g\left(x_{n}\right)$ and $h(\hat{x})=g(\hat{x})$. But this contradicts the assumption on $h$ that $\lim _{x \rightarrow \hat{x}} \frac{|h(x)-h(\hat{x})|}{(x-\hat{x})^{2}}=\infty$.

To see that $g^{-1}$ intersects only finitely many sets $D_{j}$, by way of contradiction, assume that there is an infinite set $\left\{j_{n}: n<\omega\right\}$ such that $g^{-1} \cap D_{j_{n}} \neq \emptyset$. For $n<\omega$ choose $\left\langle x_{n}, y_{n}\right\rangle \in g^{-1} \cap D_{j_{n}}$. Then $g\left(y_{n}\right)=x_{n}$ for all $n<\omega$. Choosing a subsequence, if necessary, we can assume that $\lim _{n \rightarrow \infty} x_{n}=\hat{x} \in P$. Then, $\hat{y} \stackrel{\text { def }}{=} \lim _{n \rightarrow \infty} y_{n}=\lim _{n \rightarrow \infty}\left(y_{n}-h\left(x_{n}\right)\right)+\lim _{n \rightarrow \infty} h\left(x_{n}\right)=h(\hat{x})$ and also $g(\hat{y})=\lim _{n \rightarrow \infty} g\left(y_{n}\right)=\lim _{n \rightarrow \infty} x_{n}=\hat{x}$. Since, by the assumptions from Lemma 3.2.1, $h^{\prime}(\hat{x})=0$ we obtain

$$
\begin{aligned}
1 & =\lim _{n \rightarrow \infty} \frac{g\left(y_{n}\right)-g(\hat{y})}{y_{n}-\hat{y}} \cdot \frac{y_{n}-\hat{y}}{g\left(y_{n}\right)-g(\hat{y})} \\
& =\lim _{n \rightarrow \infty} \frac{g\left(y_{n}\right)-g(\hat{y})}{y_{n}-\hat{y}} \cdot \lim _{n \rightarrow \infty} \frac{y_{n}-h(\hat{x})}{x_{n}-\hat{x}} \\
& =g^{\prime}(\hat{y}) \cdot h^{\prime}(\hat{x})=g^{\prime}(\hat{y}) \cdot 0=0,
\end{aligned}
$$

a contradiction. 
We now need to prove Lemma 3.2.1. The function $h$ constructed in the proof is a minor modification of a map $f$ from [1, thm. 18].

Proof of Lemma 3.2.1. Let $\epsilon \in(0,1)$ be such that $M<1-\epsilon$ and let $K$ be a symmetrically defined Cantor-like subset of $[0,1]$ of measure $1-\epsilon$. More precisely, the set $K$ is defined as $K=\bigcap_{n<\omega} \bigcup_{s \in 2^{n}} I_{s}=[0,1] \backslash \bigcup_{s \in 2^{<\omega}} J_{s}$, where: $2^{n}$ denotes the set of all sequences from $n=\{0,1, \ldots, n-1\}$ into $2=\{0,1\} ; 2^{<\omega}=\bigcup_{n<\omega} 2^{n}$ is the set of all finite $0-1$ sequences; $I_{\emptyset}=[0,1]$, and, for any $s \in 2^{n}, J_{s}$ is an open interval of length $\frac{\epsilon}{3^{n+1}}$ sharing the center with $I_{s}$, while $I_{s^{\wedge} 0}<I_{s^{\wedge} 1}$ are the component intervals of $I_{s} \backslash J_{s}$. Note that $\left|J_{s}\right|=\frac{\epsilon}{3^{n+1}}<\frac{1}{3^{n+1}}<\left|I_{s}\right| \leq \frac{1}{2^{n}}$ for every $s \in 2^{n}$, so the choice of $J_{s}$ is always possible. Clearly the set $K$ has the desired measure of $1-\sum_{s \in 2<\omega}\left|J_{s}\right|=$ $1-\sum_{n<\omega} 2^{n} \frac{\epsilon}{3^{n+1}}=1-\epsilon$.

For every $s \in 2^{n}$ let $f_{s}$ be a function from $\mathbb{R}$ onto $[0,1 /(n+1)]$ defined as $f_{s}(x)=\frac{2}{(n+1)\left|J_{s}\right|} \operatorname{dist}\left(x, \mathbb{R} \backslash J_{s}\right)$, where $\operatorname{dist}(x, T)=\inf \{|x-t|: t \in T\}$ denotes the distance from $x$ to $T$. Then, the function $h_{0}=\sum_{s \in 2<\omega} f_{s}: \mathbb{R} \rightarrow[0,1]$ is continuous and our $\mathcal{C}^{1}$ function $h: \mathbb{R} \rightarrow \mathbb{R}$ is defined as $h(x)=\int_{0}^{x} h_{0}(t) d t$. Note that $h$ is strictly increasing on $[0,1]$.

Let $P$ be an arbitrary perfect subset of $K$ of measure $M$, which is disjoint with the set of all endpoints of the intervals $J_{s}, s \in 2^{<\omega}$. We will show that $h$ and $P$ are as required.

Clearly, for every $\hat{x} \in P \subset K$ we have $h^{\prime}(\hat{x})=h_{0}(\hat{x})=0$. To see the other condition, first notice that for $n>1 / \ln (4 / 3)$

$$
\text { if } \hat{x}, x_{0} \in K \cap I_{s} \text { for } s \in 2^{n} \text { and } \hat{x} \neq x_{0} \text {, then } \frac{\left|h\left(x_{0}\right)-h(\hat{x})\right|}{\left(x_{0}-\hat{x}\right)^{2}} \geq \frac{\epsilon}{6} \frac{(4 / 3)^{n}}{(n+1)} \text {. }
$$


To argue for (3.3), choose the largest $m<\omega$ such that $\hat{x}, x_{0} \in I_{t}$ for some $t \in 2^{m}$. Then $m \geq n, \hat{x}$ and $x_{0}$ are separated by the interval $J_{t}$, and

$\frac{\left|h\left(x_{0}\right)-h(\hat{x})\right|}{\left(x_{0}-\hat{x}\right)^{2}}=\frac{\left|\int_{\hat{x}}^{x_{0}} h_{0}(t) d t\right|}{\left(x_{0}-\hat{x}\right)^{2}} \geq \frac{\left|\int_{J_{t}} h_{0}(t) d t\right|}{\left|I_{t}\right|^{2}}=\frac{\frac{1}{2}\left|J_{t}\right| \frac{1}{(m+1)}}{\left|I_{t}\right|^{2}} \geq \frac{\frac{1}{2} \frac{\epsilon}{3^{m+1}} \frac{1}{(m+1)}}{\left(1 / 2^{m}\right)^{2}}$

Hence, $\frac{\left|h\left(x_{0}\right)-h(\hat{x})\right|}{\left(x_{0}-\hat{x}\right)^{2}} \geq \frac{\frac{1}{2} \frac{\epsilon}{3^{m+1}} \frac{1}{(m+1)}}{\left(1 / 2^{m}\right)^{2}}=\frac{\epsilon}{6} \frac{(4 / 3)^{m}}{(m+1)} \geq \frac{\epsilon}{6} \frac{(4 / 3)^{n}}{(n+1)}$, as required, where the last inequality holds, since the function $f(x)=\frac{(4 / 3)^{x}}{x+1}$ is increasing for $x>1 / \ln (4 / 3)$, having derivative $f^{\prime}(x)=\frac{(4 / 3)^{x}[\ln (4 / 3)(x+1)-1]}{(x+1)^{2}}$.

Next, notice that

if $s \in 2^{n}, x \in J_{s}$, and $x_{0}$ is an endpoint of $J_{s}$, then $\frac{\left|h(x)-h\left(x_{0}\right)\right|}{\left(x-x_{0}\right)^{2}} \geq \frac{3^{n+1}}{4(n+1) \epsilon}$.

To argue for (3.4), let $x_{1}$ be the midpoint between $x_{0}$ and $x$. Then $h_{0}$ is linear on the interval between $x_{0}$ and $x_{1}$ with the slope $\pm \frac{2}{(n+1)\left|J_{s}\right|}$. Hence, indeed,

$$
\frac{\left|h(x)-h\left(x_{0}\right)\right|}{\left(x-x_{0}\right)^{2}}>\frac{\left|h\left(x_{1}\right)-h\left(x_{0}\right)\right|}{4\left(x_{1}-x_{0}\right)^{2}}=\frac{\frac{1}{2}\left(x_{1}-x_{0}\right)^{2} \frac{2}{(n+1)\left|J_{s}\right|}}{4\left(x_{1}-x_{0}\right)^{2}}=\frac{3^{n+1}}{4(n+1) \epsilon} .
$$

Finally, fix an $\hat{x} \in P$. We need to show that $\lim _{x \rightarrow \hat{x}} \frac{|h(x)-h(\hat{x})|}{(x-\hat{x})^{2}}=\infty$. For this, we fix an arbitrarily large $N$ and show that $\frac{|h(x)-h(\hat{x})|}{(x-\hat{x})^{2}} \geq N$ for the points $x$ close enough to $\hat{x}$.

Let $n_{0}$ be such that $\min \left\{\frac{\epsilon}{6} \frac{(4 / 3)^{n}}{(n+1)}, \frac{3^{n+1}}{4(n+1) \epsilon}\right\} \geq 4 N$ for all $n \geq n_{0}$ and let $s \in 2^{n_{0}}$ be such that $\hat{x} \in I_{s}$. Notice that $\hat{x}$ belongs to the interior $U$ of $I_{s}$, as $\hat{x} \in P$. Hence, it is enough to show that $\frac{|h(x)-h(\hat{x})|}{(x-\hat{x})^{2}} \geq N$ for every $x \neq \hat{x}$ from $U$. So, fix such an $x$. 
If $x \in K$, then $\frac{|h(x)-h(\hat{x})|}{(x-\hat{x})^{2}} \geq N$ follows immediately from (3.3). So, assume that $x \notin K$. Then $x \in J_{t}$ for some $t \supset s$. Let $x_{0}$ be the end point of $J_{t}$ between $x$ and $\hat{x}$. Notice, that $x_{0} \neq \hat{x}$, since $\hat{x} \in P$. Then, since $h$ is increasing on $[0,1]$, properties (3.3) and (3.4) imply

$$
\begin{aligned}
\frac{|h(x)-h(\hat{x})|}{(x-\hat{x})^{2}} & =\frac{\left|h(x)-h\left(x_{0}\right)\right|}{\left(x-x_{0}\right)^{2}} \frac{\left(x-x_{0}\right)^{2}}{(x-\hat{x})^{2}}+\frac{\left|h\left(x_{0}\right)-h(\hat{x})\right|}{\left(x_{0}-\hat{x}\right)^{2}} \frac{\left(x_{0}-\hat{x}\right)^{2}}{(x-\hat{x})^{2}} \\
& \geq 4 N \frac{\left(x-x_{0}\right)^{2}}{(x-\hat{x})^{2}}+4 N \frac{\left(x_{0}-\hat{x}\right)^{2}}{(x-\hat{x})^{2}} \geq N
\end{aligned}
$$

finishing the proof.

\subsection{The Second Construction}

In this section, we give a simpler construction of a $\mathfrak{C}\left(D^{2}\right)$-continuous function $f: \mathbb{R}^{2} \rightarrow \mathbb{R}$ such that $D(f)$ is a perfect set. In order to construct our function, we will need the following lemma proved as [15, Example 4.5.1].

Lemma 3.3.1 There exists a homeomorphism $h: \mathbb{R} \rightarrow \mathbb{R}$ and a perfect set $P \subset \mathbb{R}$ such that $h$ and $h^{-1}$ are "C ${ }^{2}$ " and $h \uparrow P$ is identically infinite.

Reall that " $\mathrm{C}^{2}$ " is the class of functions with continuous second derivative, where we allow the seond derivative to assume infinte values. Proceding much the same way we did in the first section, we will construct a $\mathfrak{C}\left(\mathcal{D}^{2}\right)$-continuous function whose discontinuity set will coincide with $h\lceil P$.

Theorem 3.3.2 Let $h$ and $P$ be as in Lemma 3.3.1. Then there is a $\mathfrak{C}\left(\mathcal{D}^{2}\right)$ continuous function $F: \mathbb{R}^{2} \rightarrow \mathbb{R}$ with $D(F)=h \uparrow P$. 
Proof. Let $\left\{J_{j}: j<\omega\right\}$ be an enumeration, without repetitions, of bounded connected components of $\mathbb{R} \backslash P$. For every $j<\omega$ let the $I_{j}$ be the open middle third subinterval of $J_{j}$ and let $F_{j}$ be a continuous function from $\mathbb{R}^{2}$ onto $[0,1]$ with $\operatorname{supp}\left(F_{j}\right)$ contained in some rectangle $S_{j} \subset D_{j} \stackrel{\text { def }}{=}\left\{\langle x, y\rangle \in \mathbb{R}^{2}: x \in\right.$ $\left.I_{j} \&|y-h(x)|<\left|I_{j}\right|^{3}\right\}$, where $\left|I_{j}\right|$ is the length of $I_{j}$. Moreover, choose $S_{j}$ such that $\left|\pi_{x}\left(S_{j}\right)\right|<\left|\pi_{y}\left(S_{j}\right)\right|^{3}$, where $\pi_{x}$ and $\pi_{y}$ denote the projection maps onto the $x$ and $y$ axes, respectivley. We will show that the function $F=\sum_{j<\omega} F_{j}$ is as required.

It is enough to show that sets $S_{j}$ satisfy the property (F) from Lemma 2.3.1 for $\mathcal{D}^{2} \cup\left(\mathcal{D}^{2}\right)^{-1}$. To see this, fix a $\mathcal{D}^{2}$ function $g: \mathbb{R} \rightarrow \mathbb{R}$. We need to prove that both $g$ and $g^{-1}$ intersect only finitely many sets $D_{j}$, hence only finitely many of the sets $S_{j}$.

To see that $g$ intersects only finitely many sets $D_{j}$, by way of contradiction, assume that there is an infinite set $\left\{j_{n}: n<\omega\right\}$ such that $g \cap D_{j_{n}} \neq \emptyset$. For $n<\omega$ choose $\left\langle x_{n}, y_{n}\right\rangle \in g \cap D_{j_{n}}$. Then $g\left(x_{n}\right)=y_{n}$ for all $n<\omega$. Choosing a subsequence, if necessary, we can assume that $\lim _{n \rightarrow \infty} x_{n}=\hat{x} \in P$. Then, by the definition of sets $D_{j}, \lim _{n \rightarrow \infty}\left|y_{n}-h\left(x_{n}\right)\right| \leq \lim _{n \rightarrow \infty}\left|I_{j_{n}}\right|^{3}=0$, and

$$
g(\hat{x})=\lim _{n \rightarrow \infty} g\left(x_{n}\right)=\lim _{n \rightarrow \infty} y_{n}=\lim _{n \rightarrow \infty}\left(y_{n}-h\left(x_{n}\right)\right)+\lim _{n \rightarrow \infty} h\left(x_{n}\right)=h(\hat{x}) .
$$

So, since $\lim _{n \rightarrow \infty}\left|\frac{y_{n}-h\left(x_{n}\right)}{x_{n}-\hat{x}}\right| \leq \lim _{n \rightarrow \infty} \frac{\left|y_{n}-h\left(x_{n}\right)\right|}{\left|I_{k_{n}}\right|} \leq \lim _{n \rightarrow \infty}\left|I_{k_{n}}\right|^{2}=0$,

$$
g^{\prime}(\hat{x})=\lim _{n \rightarrow \infty} \frac{y_{n}-h(\hat{x})}{x_{n}-\hat{x}}=\lim _{n \rightarrow \infty} \frac{y_{n}-h\left(x_{n}\right)}{x_{n}-\hat{x}}+\lim _{n \rightarrow \infty} \frac{h\left(x_{n}\right)-h(\hat{x})}{x_{n}-\hat{x}}=h^{\prime}(\hat{x}) .
$$


This is the first major difference between the proof of Theorem 3.3.2 and the proof of Theorem 3.2.2. Here, although for the occasional $\hat{x}, h(\hat{x})$ may equal 0 , this can happen at most finitely many times. So, we must proceed a little differently from here out. This is also the source of the need for the more complicated generalized second derivative. Notice that for any function $f$ which has a second derivative at $\hat{x}$, we have, using L'Hôpital's rule

$$
\begin{aligned}
\lim _{x \rightarrow \hat{x}} \frac{f(x)-\left[f^{\prime}(\hat{x})(x-\hat{x})+f(\hat{x})\right]}{(x-\hat{x})^{2}} & =\lim _{x \rightarrow \hat{x}} \frac{f^{\prime}(x)-f^{\prime}(\hat{x})}{2(x-\hat{x})} \\
& =\frac{1}{2} f^{\prime \prime}(x) .
\end{aligned}
$$

So $\lim _{n \rightarrow \infty} \frac{h\left(x_{n}\right)-\left[h^{\prime}(\hat{x})\left(x_{n}-\hat{x}\right)+h(\hat{x})\right]}{\left(x_{n}-\hat{x}\right)^{2}}$ should be equal to $\infty$ for every $\hat{x} \in P$. Similarly, $\lim _{n \rightarrow \infty} \frac{g\left(x_{n}\right)-\left[g^{\prime}(\hat{x})\left(x_{n}-\hat{x}\right)+g(\hat{x})\right]}{\left(x_{n}-\hat{x}\right)^{2}}=\frac{1}{2} g^{\prime \prime}(\hat{x})$.

But since

$$
\lim _{n \rightarrow \infty}\left|\frac{h\left(x_{n}\right)-y_{n}}{\left(x_{n}-\hat{x}\right)^{2}}\right| \leq \lim _{n \rightarrow \infty} \frac{\left|y_{n}-h\left(x_{n}\right)\right|}{\left|I_{k_{n}}\right|^{2}} \leq \lim _{n \rightarrow \infty}\left|I_{k_{n}}\right|=0
$$

we get

$$
\begin{aligned}
\lim _{n \rightarrow \infty} \frac{h\left(x_{n}\right)-\left[h^{\prime}(\hat{x})\left(x_{n}-\hat{x}\right)+h(\hat{x})\right]}{\left(x_{n}-\hat{x}\right)^{2}} & =\lim _{n \rightarrow \infty}\left[\frac{h\left(x_{n}\right)-y_{n}}{\left(x_{n}-\hat{x}\right)^{2}}+\frac{y_{n}-h(\hat{x})}{\left(x_{n}-\hat{x}\right)^{2}}+\frac{h^{\prime}(\hat{x})}{x_{n}-\hat{x}}\right] \\
& =\lim _{n \rightarrow \infty}\left[\frac{h\left(x_{n}\right)-y_{n}}{\left(x_{n}-\hat{x}\right)^{2}}+\frac{g^{\prime}(\hat{x})\left(x_{n}-\hat{x}\right)+g(\hat{x})}{\left(x_{n}-\hat{x}\right)^{2}}\right] \\
& =\frac{1}{2} g^{\prime \prime}(\hat{x})
\end{aligned}
$$

which is clearly finite. Thus we have a contradiction. So no twice differentiable curve $g$ can intersect infinitely many disks. 
We now need to deal with the claim that the inverse of a twice differentiable function cannot intersect infinitely many of the disks $D_{i}$. While the proof from the paper holds so far as showing that $\left|\frac{1}{g^{\prime}(\hat{y})}\right|=\left|h^{\prime}(x)\right|$, we can no longer equate this value with 0 . In fact, this can only hold for at most finitely many $\hat{x} \in P$. So that argument is no longer a valid way of showing that $g^{-1}$ intersects at most finitely many disks.

We must essentially repeat our argument for $g$, this time considering how many of the smaller sets $S_{j}$ the set $g^{-1}$ can intersect. So, we assume that there is an infinite set $\left\{j_{n}: n<\omega\right\}$ such that $g^{-1} \cap D_{j_{n}} \neq \emptyset$. For $n<\omega$, choose $\left\langle x_{n}, y_{n}\right\rangle \in g^{-1} \cap S_{j_{n}}$. Then $g\left(y_{n}\right)=x_{n}$ for all $n<\omega$. Choosing a subsequence, if necessary, we may assume that $\lim _{n \rightarrow \infty}\left\langle x_{n}, y_{n}\right\rangle=\langle\hat{x}, \hat{y}\rangle$, with $\hat{x} \in P$. Then,

$$
\hat{y}=\lim _{n \rightarrow \infty} y_{n}=\lim _{n \rightarrow \infty}\left(y_{n}-h\left(x_{n}\right)\right)+\lim _{n \rightarrow \infty} h\left(x_{n}\right)=h(\hat{x}) .
$$

So $g(\hat{y})=\lim _{n \rightarrow \infty} g\left(y_{n}\right)=\lim _{n \rightarrow \infty} x_{n}=\hat{x}$.

Proceding as before, we see that since

$$
\lim _{n \rightarrow \infty}\left|\frac{x_{n}-h^{-1}\left(y_{n}\right)}{y_{n}-\hat{y}}\right| \leq \lim _{n \rightarrow \infty} \frac{\left|x_{n}-h^{-1}\left(y_{n}\right)\right|}{\left|\pi_{y}\left(S_{j_{n}}\right)\right|} \leq \lim _{n \rightarrow \infty}\left|\pi_{y}\left(S_{j_{n}}\right)\right|^{2}=0,
$$

where the second inequality is due to the estimate we required on the size of the projections of the sets $S_{j}$. We now have

$g^{\prime}(\hat{y})=\lim _{n \rightarrow \infty} \frac{x_{n}-h^{-1}(\hat{y})}{y_{n}-\hat{y}}=\lim _{n \rightarrow \infty} \frac{x_{n}-h\left(y_{n}\right)}{y_{n}-\hat{y}}+\lim _{n \rightarrow \infty} \frac{h^{-1}\left(y_{n}\right)-h^{-1}(\hat{y})}{y_{n}-\hat{y}}=\left(h^{-1}\right)^{\prime}(\hat{y})$. 
Since

$$
\lim _{n \rightarrow \infty}\left|\frac{x_{n}-h^{-1}\left(y_{n}\right)}{\left(y_{n}-\hat{y}\right)^{2}}\right| \leq \lim _{n \rightarrow \infty} \frac{\left|x_{n}-h^{-1}\left(y_{n}\right)\right|}{\pi_{y}\left(S_{j_{n}}\right)^{2}} \leq \lim _{n \rightarrow \infty}\left|\pi_{y}\left(S_{j_{n}}\right)\right|=0
$$

we have the following.

$$
\begin{aligned}
& \lim _{n \rightarrow \infty} \frac{h^{-1}\left(y_{n}\right)-\left[\left(h^{-1}\right)^{\prime}(\hat{y})\left(y_{n}-\hat{y}\right)+h^{-1}(\hat{y})\right]}{\left(y_{n}-\hat{y}\right)^{2}}= \\
= & \lim _{n \rightarrow \infty}\left[\frac{h^{-1}\left(y_{n}\right)-x_{n}}{\left(y_{n}-\hat{y}\right)^{2}}+\frac{x_{n}-h^{-1}(\hat{y})}{\left(y_{n}-\hat{y}\right)^{2}}-\frac{\left(h^{-1}\right)^{\prime}(\hat{y})}{y_{n}-\hat{y}}\right] \\
= & \lim _{n \rightarrow \infty}\left[\frac{h^{-1}\left(y_{n}\right)-x_{n}}{\left(y_{n}-\hat{y}\right)^{2}}+\frac{g\left(y_{n}\right)-g(\hat{y})}{\left(y_{n}-\hat{y}\right)^{2}}-\frac{g^{\prime}(\hat{y})}{y_{n}-\hat{y}}\right] \\
= & \lim _{n \rightarrow \infty}\left[\frac{h^{-1}\left(y_{n}\right)-x_{n}}{\left(y_{n}-\hat{y}\right)^{2}}+\frac{g\left(y_{n}\right)-\left[g^{\prime}(\hat{y})\left(y_{n}-\hat{y}\right)\right]+g(\hat{y})}{\left(y_{n}-\hat{y}\right)^{2}}\right] \\
= & \lim _{n \rightarrow \infty} \frac{g^{\prime}\left(y_{n}\right)-g^{\prime}(\hat{y})}{2\left(y_{n}-\hat{y}\right)}=\frac{1}{2} g^{\prime \prime}(\hat{x})
\end{aligned}
$$

Since $g^{-1} \in \mathcal{C}^{2}$, we know that this quantity is nonzero. But, since $h(\hat{x})=$ $\infty$ then $h^{-1}(\hat{y})=0$. Thus, we have reached a contradiction. Thus we see that no $\mathcal{C}^{2}$ function $g$ can intersect infintely many of the sets, $S_{j}$.

\subsection{Discussion}

The two constructions described in this chapter cannot be extended to create $\mathfrak{C}\left(\mathcal{D}^{2}\right)$-continuous functions defined on $\mathbb{R}^{n}$ for $n>2$, being as in this dimension, $\mathfrak{C}\left(\mathcal{D}^{2}\right)$ can not be written as $\mathcal{D}^{2} \cup\left(\mathcal{D}^{2}\right)^{-1}$. In fact, it is not even clear what such an expression would mean. Furthermore, as long as we keep the 
definition of "smooth curve" mentioned in Chapter 1, we cannot use a trick similar to Hahn's, that is allowing $F(x, y)$ to be a $\mathfrak{C}\left(\mathcal{D}^{2}\right)$-continuous function for which $\operatorname{dim}_{\mathcal{H}}(D(F))=1$, and then define $\phi\left(x_{1}, x_{2}, \ldots, x_{n}\right)=F\left(x_{1}, x_{2}\right)$. This function would not necessarily be $\mathfrak{C}\left(\mathcal{D}^{2}\right)$-continuous. Consider any curve $\alpha=\left\langle\alpha_{1}(t), \alpha_{2}(t), \ldots, \alpha_{n}(t)\right\rangle$ with the property that $\alpha_{i}(t)>0$ for all $t$ and all $i \geq 3$, but for which there is a point $t_{0}$ for which $\alpha_{1}\left(t_{0}\right)=\alpha_{2}\left(t_{0}\right)=0$. Then, even though, the curve defined by $\alpha$ is $\mathcal{D}^{2}, \phi\lceil\alpha([0,1])$ may fail to be continuous, being as the projection of the curve into the $\left\langle x_{1}, x_{2}\right\rangle$-plane would not be smooth at $t_{0}$, and so $F$ would not be continuous on its image. 


\section{Chapter 4}

\section{Sufficient Conditions for Membership in $\mathcal{D}_{1, n}$.}

\subsection{Introduction}

Following closely, the paper [10], we take up the study of linearly continuous functions in this chapter. These functions have recieved the attention of many important mathematicians since the end of the ninteenth century. Theorem 1.3.7 has had a sweeping influence on the theory of linearly continuous functions. One of the most significant consequences of Theorem 1.3.7 is that the structure of the sets in $\mathcal{D}_{1, n}$ is very different from that of the sets in $\mathcal{D}_{1, n}^{+}$. $\mathcal{D}_{1, n}^{+}$requires only that the orthogonal projections onto coordinate hyperplanes be meager; however, even having every orthogonal projection meager is not enough to guarantee that a set is in $\mathcal{D}_{1, n}$ (see [45]). Nevertheless, every such projection is meager. As has already been noted, if $f: \mathbb{R}^{n} \rightarrow \mathbb{R}$ is linearly continuous, then $\operatorname{dim}_{\mathcal{H}}(D(f)) \leq n-1$. Furthermore, 
most of the constructions of linearly continuous functions have been in $\mathbb{R}^{2}$ and were constructed without regard to having large discontinuity set in the sense of measure.

The main results we give in this section pertain to the discontinuity sets of linearly continuous functions. Given Theorem 1.3.7, it seems natural to look for sufficient conditions for membership in $\mathcal{D}_{1, n}$ by asking what types of Lipschitz functions on a nowhere dense subset of a hyperplane can be the set of discontinuity points of a linearly continuous function. We provide two such sufficient conditions, one of which is valid for only for $\mathbb{R}^{2}$, whereas the other is valid for $\mathbb{R}^{n}$ for all $n$. We then procede to discuss the degree to which Theorem 1.3.1 can be extended to linearly continuous functions.

For this section, in our proofs, we use a few extra symbols which do not occur elsewhere. For a class $\mathcal{F}$ of (possibly partial) functions from $\mathbb{R}^{n-1}$ into $\mathbb{R}$, let $\mathcal{E}(\mathcal{F})$ be the collection of all sets $D=\bigcup_{i=1}^{\infty} D_{i}$ where each $D_{i}$ is isometric to $\phi\left\lceil K\right.$ for some $\phi \in \mathcal{F}$ and a compact nowhere dense $K \subset \mathbb{R}^{n-1}$. Using this notation, we could restate Theorem 1.3.7 as

$$
\mathcal{D}_{1, n} \subset \mathcal{E}\left(\operatorname{Lip}\left(\mathbb{R}^{n-1}\right)\right) .
$$

Recall that every partial Lipschitz function on a subset of $\mathbb{R}^{n}$ can be extended to a Lipschitz function on all of $\mathbb{R}^{n}$ (see, for instance [19, p. 80] or $[26])$. Moreover, since the family $\mathcal{D}_{1, n}$ is clearly closed under isometric images (i.e., if $D \in \mathcal{D}_{1, n}$, then $i[D] \in \mathcal{D}_{1, n}$ for every isometry $i$ of $\mathbb{R}^{n}$ ) and under countable unions ${ }^{1}$, we have the following result, where $R(\mathcal{F})$ represents the

\footnotetext{
${ }^{1}$ We may fix the oscillation of $f$ on any set $D \in \mathcal{D}^{n}$ to be at most 1 and use a procedure
} 
property:

$R(\mathcal{F}): \phi \uparrow K \in \mathcal{D}_{1, n}$ for every $\phi \in \mathcal{F}$ and a compact nowhere dense $K \subset$ $\mathbb{R}^{n-1}$.

Fact 4.1.1 For every family $\mathcal{F}$ of functions from $H \subset \mathbb{R}^{n-1}$ into $\mathbb{R}$, if $R(\mathcal{F})$ holds, then $\mathcal{E}(\mathcal{F}) \subset \mathcal{D}_{1, n}$.

We will make extensive use of this fact; it is vital for the proof of the central resutl of this chapter.

The main result of the next two sections is as follows, where the symbols $\operatorname{Conv}\left(\mathbb{R}^{n}\right)$ and $\mathcal{C}^{2}\left(\mathbb{R}^{n}\right)$ stand for all functions $\phi: \mathbb{R}^{n} \rightarrow \mathbb{R}$ that are, respectively, convex and continuously twice differentiable.

Theorem 4.1.2 The property $R(\mathcal{F})$ holds for $\mathcal{F}=\operatorname{Conv}\left(\mathbb{R}^{n-1}\right)$ for any $n \geq 2$ and for $\mathcal{F}=\mathcal{C}^{2}\left(\mathbb{R}^{1}\right)$.

We will prove this theorem later in this section, but first, we discuss some of its consequences. Concerning its relation to the literature, we note that this result will be contrasted to [44, Section 5], where it is asserted, without proof, that any nowhere dense subset of a "smooth curve" is $D(f)$ for some linearly continuous $f: \mathbb{R}^{2} \rightarrow \mathbb{R}$. Note, however, our Proposition 4.1.4, which shows that general smooth curves, using our definition, do not have this property.

Corollary 4.1.3 For every $n \geq 2$

(a) $\mathcal{E}\left(\operatorname{Conv}\left(\mathbb{R}^{n-1}\right)\right) \subsetneq \mathcal{D}_{1, n} \subsetneq \mathcal{E}\left(\operatorname{Lip}\left(\mathbb{R}^{n-1}\right)\right)$, similar to what is found in [35, pp. 31-32]. 
(b) $\mathcal{E}\left(\mathcal{C}^{2}(\mathbb{R})\right) \subsetneq \mathcal{D}_{1,2} \subsetneq \mathcal{E}(\operatorname{Lip}(\mathbb{R}))$.

Proof. The inclusions follow from Theorem 4.1.2, Fact 4.1.1, and Theorem 1.3.7.

To see that they are all strict, first notice that, clearly, neither of the classes $\mathcal{C}^{2}(\mathbb{R})$ and $\operatorname{Conv}(\mathbb{R})$ contains the other. Actually, if $\Phi: \mathbb{R} \rightarrow \mathbb{R}$ is a convex function with a (countable) dense set $A$ of points at which $\Phi^{\prime \prime}$ does not exist (see e.g. [42]) and $K$ is a nowhere dense perfect set for which the set $A_{0}=\{a \in A: a$ is a bilateral limit point of $K\}$ is dense in $K$, then $f\lceil K \in$ $\mathcal{E}(\operatorname{Conv}(\mathbb{R})) \backslash \mathcal{E}\left(\mathcal{C}^{2}(\mathbb{R})\right)$. It is also possible to find a $\mathcal{C}^{\infty}$ function $\Phi: \mathbb{R} \rightarrow \mathbb{R}$ and a nowhere dense perfect set $K \subset \mathbb{R}$ for which $f \nmid K \notin \mathcal{E}(\operatorname{Conv}(\mathbb{R}))$. Therefore, the first inclusions in (a) and (b) are indeed strict (at least, for $n=2)$.

The fact that the remaining inclusions are strict follows from Proposition 4.1.4.

Notice that if we let $K \subset \mathbb{R}^{n-1}$ be an $(n-2)$-flat, then the constant function $\hat{c}: K \rightarrow \mathbb{R}$ given by $\hat{c}(x)=c$ is convex. The graph of this function is an $(n-2)$-flat in $\mathbb{R}^{n}$. Since $K$ is nowhere dense in $\mathbb{R}^{n-1}$, we have shown, in particular, that linearly continuous functions may be discontinuous at every point of an $(n-2)$-flat.

It should be noted that in spite of the pleasing results mentioned so far, not all "small" subsets of curves can be the sets of discontinuities of a linearly continuous function. In particular, let $b D^{1}\left(\mathbb{R}^{n}\right)$ stand for the class of all differentiable functions $\phi: \mathbb{R}^{n} \rightarrow \mathbb{R}$ with bounded derivative. Notice that $b D^{1}\left(\mathbb{R}^{n}\right) \subset \operatorname{Lip}\left(\mathbb{R}^{n}\right)$. 
Proposition 4.1.4 There exists a differentiable function $\phi: \mathbb{R}^{n-1} \rightarrow \mathbb{R}$ and a nowhere dense perfect set $K \subset \mathbb{R}^{n-1}$ such that $\phi \uparrow K \notin \mathcal{D}_{1, n}^{+}$. In particular, $\mathcal{E}\left(b D^{1}\left(\mathbb{R}^{n-1}\right)\right)$ is contained in none of the classes $\mathcal{E}(\mathcal{F})$ from Corollary 4.1.3.

Proof. First, we prove this for $n=2$. Let $K \subset[0,1]$ be nowhere dense of positive measure. By Kershner's result, Theorem 1.3.6, it is enough to find a function $\phi: \mathbb{R} \rightarrow \mathbb{R}$ from $b D^{1}(\mathbb{R})$ for which $\phi[K]$ has non-empty interior (as $\phi[K]$ is a projection of $\phi \uparrow K$ along the $x$-axis). The easiest example of such a function is given by $\phi(x)=\int_{0}^{x} g(t) d t$ for an appropriate function $g: \mathbb{R} \rightarrow$ $[0,1]$. Notice, that $g=\chi_{K}$, the characteristic function of $K$, has almost all these properties: it maps $K$ onto a non-empty interval and resulting $\phi$ has bounded derivative almost everywhere. To insure that $\phi$ is actually everywhere differentiable, it is enough to take as $g: \mathbb{R} \rightarrow[0,1]$ a nonnegative approximately continuous function with $\{x \in \mathbb{R}: g(x)>0\}$ being a subset of $K$ of positive measure. Such a function may be constructed, by allowing $U$ to be the density interior of $K$ (i.e., the set of all points of $K$ with density 1 ) and by choosing a point $p \in U$ and setting $g(p)=1$ and using the regularity of the density topology to extend $g$ to the unit interval in such a way that $g$ identically vanishes outside of $U$. That $\{x: g(x)>0\}$ has positive measure follows from the fact that as $g$ is approximately continuous, it is continuous with respect to the density topology; that is, $g^{-1}(O)$ is density open for all open sets $O$, hence $g^{-1}((0,1))$ must have positive measure. Then $\phi[K]$ still has a non-empty interior and $\phi^{\prime}(x)=g(x)$ for all $x \in \mathbb{R}$. (For more on approximately continuous functions, as well as the density topology, see e.g. [5] or [13].)

For $n>2$, let $K$ be as above and put $\hat{K}=K \times[0,1]^{n-2}$. Define 
$\phi_{n}\left(x_{1}, x_{2}, \ldots, x_{n-1}\right)=\phi\left(x_{1}\right)$. Then the projection $\pi_{x_{1}}[\phi \uparrow K]$ has nonempty interior.

This shows us that Theorem 1.3.7 is not a characterization of the sets of discontinuity of linearly continuous functions. However, the above discussed result, Corollary 4.1.3 shows that it is, in fact, quite close: Theorem 1.3.7 remains true if the causality is reversed and the word "Lipschitz" is replaced with the word "convex".

\subsection{A Sufficient Condition for Membership in $\mathcal{D}_{1, n}$}

This section will culminate in a proof of half of the promised results, namely that $R\left(\operatorname{Conv}\left(\mathbb{R}^{n-1}\right)\right)$ holds. Our construction will be based on the following simple general observation, a simple restatement of Lemma 2.3.1.

Lemma 4.2.1 Let $P \subset \mathbb{R}^{n}$ be compact nowhere dense and let

(*) $\left\{B_{i}: i<\omega\right\}$ be a family of pairwise disjoint closed balls in $\mathbb{R}^{n}$ disjoint with $P$, with non-empty interiors, and such that the closure of $\bigcup_{i<\omega} B_{i}$ is equal to $P \cup \bigcup_{i<\omega} B_{i}$.

For every $i<\omega$, let $f_{i}$ be a continuous function from $\mathbb{R}^{n}$ onto $[0,1]$ with the support contained in $B_{i}$. Then $f=\sum_{i<\omega} f_{i}$ is from $\mathbb{R}^{n}$ onto $[0,1]$ and

(a) $D(f)=P=\left\{x \in \mathbb{R}^{n}: \operatorname{osc}(f, x)=1\right\}$.

Moreover, if 
(b) every line in $\mathbb{R}^{n}$ intersects only finitely many balls $B_{i}$, then $f$ is linearly continuous.

Proof. The result is obvious, after one notices that every open set intersecting $P$ fully contains one of the balls $B_{i}$. (This can be additionally imposed in the assumptions, but in $\mathbb{R}^{n}$ it actually is already ensured by the current assumptions.)

All linearly continuous functions we construct here will be of the form of a function $f$ from Lemma 4.2.1. Clearly, we will use this with $P$ being $\phi\lceil K$ for appropriate $\phi$ and $K$. The construction of the balls satisfying $(*)$ is an easy exercise. It is the property (b) that will require care.

We will also need the following simple result.

Lemma 4.2.2 Let $\hat{B}_{i}$ 's and $\hat{P} \subset \mathbb{R}^{n-1}$ be as in Lemma 4.2.1. Then there exists a $\mathcal{C}^{2}$ function $h: \mathbb{R}^{n-1} \rightarrow[0,1]$ such that $h(x)>0$ if, and only if, $x$ belongs to the interior of one of the balls $\hat{B}_{i}$.

Proof. Actually, such a function can be even $\mathcal{C}^{\infty}$. Simply, for any $i<$ $\omega$ choose a $C^{\infty}$ function $h_{i}: \mathbb{R}^{n-1} \rightarrow[0,1]$ for which $\left\{x \in \mathbb{R}^{n-1}: h_{i}(x)>\right.$ $0\}$ is the interior of the ball $\hat{B}_{i}$. Then, for appropriately chosen numbers $a_{i}>0$ (with the sequence $\left\langle a_{i}\right\rangle_{i}$ converging quickly to 0 ), the function $h=$ $\sum_{i<\omega} a_{i} h_{i}$ is $\mathcal{C}^{\infty}$ and as required. (For similar constructions of $\mathcal{C}^{\infty}$ functions, see also [14].)

We will use one more lemma. 
Lemma 4.2.3 Let $\phi: \mathbb{R}^{n-1} \rightarrow \mathbb{R}$ be an arbitrary continuous function, $\hat{P} \subset$ $\mathbb{R}^{n-1}$ be closed nowhere dense, and let $h$ and the $\hat{B}_{i}$ 's be as in Lemma 4.2.2. For every $i<\omega$ choose an arbitrary closed ball $B_{i}$ inside the set

$$
T_{i}=\left\{\langle x, y\rangle: x \in \hat{B}_{i} \& y \in(\phi(x),(\phi+h)(x))\right\}
$$

In particular, each $B_{i}$ is strictly above the graph of $\phi$. Then, for every $N>0$ and line $\ell$ in $\mathbb{R}^{n}$ if the set $\left\{i<\omega: \ell \cap B_{i} \cap[-N, N]^{n} \neq \emptyset\right\}$ is infinite, then

(i) $\ell$ is non-vertical, so it can be identified with a function $L$ from a line $\ell_{0}$ in $\mathbb{R}^{n-1}$ into $\mathbb{R}$,

(ii) there is a sequence $\left\langle b_{i_{k}} \in \ell_{0} \cap \hat{B}_{i_{k}}: k<\omega\right\rangle$ converging monotonically on $\ell_{0}$ to a $b \in \ell_{0} \cap \hat{P}$ and such that: $\phi(b)=L(b)$ and $\lim _{k \rightarrow \infty} \frac{\phi\left(b_{i_{k}}\right)-\phi(b)}{\left\|b_{i_{k}}-b\right\|}$ exists and is equal $\lim _{k \rightarrow \infty} \frac{L\left(b_{i_{k}}\right)-L(b)}{\left\|b_{i_{k}}-b\right\|}$, the slope of the line $L: \ell_{0} \rightarrow \mathbb{R}$ when $\ell_{0}$ is oriented in such a way, that points $b_{i_{k}}$ are to the right of $b$.

Proof. Clearly $\ell$ cannot be vertical, since each $B_{i}$ is a subset of the set $T_{i}=\left\{\langle x, y\rangle: x \in \hat{B}_{i} \& y \in(\phi(x),(\phi+h)(x))\right\}$ and $\hat{B}_{i}$ 's are pairwise disjoint. So, there is an $\ell_{0}$ as in (i) and we can choose a sequence $\left\langle b_{i_{k}}\right\rangle_{k<\omega}$ of points in $\ell_{0}$, each from a different ball $\hat{B}_{i_{k}}$, with $\left\langle b_{i_{k}}, L\left(b_{i_{k}}\right)\right\rangle \in B_{i_{k}} \cap[-N, N]^{n}$ for every $k<\omega$. By $(*)$, choosing a subsequence, if necessary, we can assume that $\left\langle b_{i_{k}}\right\rangle_{k<\omega}$ converges monotonically on $\ell_{0}$ to some $b \in \hat{P} \cap \ell_{0}$. So, $L(b)=$ $\lim _{k \rightarrow \infty} L\left(b_{i_{k}}\right)$ and $\phi(b)=\lim _{k \rightarrow \infty} \phi\left(b_{i_{k}}\right)$.

The rest is a consequence of the squeeze theorem. Indeed, for every $k<\omega$ we have $\phi\left(b_{i_{k}}\right) \leq L\left(b_{i_{k}}\right) \leq(\phi+h)\left(b_{i_{k}}\right)=\phi\left(b_{i_{k}}\right)+h\left(b_{i_{k}}\right)$. Hence, taking limit over $k \rightarrow \infty$, we get $\phi(b) \leq L(b) \leq \phi(b)+h(b)=\phi(b)$, where $h(b)=0$ 
follows from the fact that $b \in \hat{P}$ does not belong to any interior of a ball $\hat{B}_{i}$. Similarly,

$$
\lim _{k \rightarrow \infty} \frac{h\left(b_{i_{k}}\right)-h(b)}{\left\|b_{i_{k}}-b\right\|}=0,
$$

since the limit equals the directional derivative $D_{\vec{u}} h(b)$ of $h$ at $b$ in the direction of the line $\ell_{0}$ and $D_{\vec{u}} h(b)=0$, since between any $b_{i+k}$ and $b$ there is a $c_{i_{k}} \in \ell_{0}$ (from the boundary of $\hat{B}_{i_{k}}$ ) with $h\left(c_{i_{k}}\right)=0$.

Finally, for every $k<\omega$ we have $L\left(b_{i_{k}}\right)-h\left(b_{i_{k}}\right) \leq \phi\left(b_{i_{k}}\right) \leq L\left(b_{i_{k}}\right)$, so

$$
\begin{aligned}
\frac{L\left(b_{i_{k}}\right)-L(b)}{\left\|b_{i_{k}}-b\right\|}-\frac{h\left(b_{i_{k}}\right)-h(b)}{\left\|b_{i_{k}}-b\right\|} & =\frac{\left(L\left(b_{i_{k}}\right)-h\left(b_{i_{k}}\right)\right)-\phi(b)}{\left\|b_{i_{k}}-b\right\|} \\
& \leq \frac{\phi\left(b_{i_{k}}\right)-\phi(b)}{\left\|b_{i_{k}}-b\right\|} \leq \frac{L\left(b_{i_{k}}\right)-L(b)}{\left\|b_{i_{k}}-b\right\|}
\end{aligned}
$$

and, taking limit over $k \rightarrow \infty$, we get

$$
\lim _{k \rightarrow \infty} \frac{L\left(b_{i_{k}}\right)-L(b)}{\left\|b_{i_{k}}-b\right\|}-0 \leq \lim _{k \rightarrow \infty} \frac{\phi\left(b_{i_{k}}\right)-\phi(b)}{\left\|b_{i_{k}}-b\right\|} \leq \lim _{k \rightarrow \infty} \frac{L\left(b_{i_{k}}\right)-L(b)}{\left\|b_{i_{k}}-b\right\|}
$$

and the desired equation $\lim _{k \rightarrow \infty} \frac{\phi\left(b_{i_{k}}\right)-\phi(b)}{\left\|b_{i_{k}}-b\right\|}=\lim _{k \rightarrow \infty} \frac{L\left(b_{i_{k}}\right)-L(b)}{\left\|b_{i_{k}}-b\right\|}$ holds.

These lemmas suffice for our desired result.

Proof of Theorem 4.1.2 For $\mathcal{F}=\operatorname{Conv}\left(\mathbb{R}^{n-1}\right)$. Let $\hat{P} \subset \mathbb{R}^{n-1}$ be compact nowhere dense and $\phi: \mathbb{R}^{n-1} \rightarrow \mathbb{R}$ be a convex. So, $\phi$ is continuous (see [40]). We need to show that $\phi \uparrow \hat{P} \in \mathcal{D}_{1, n}$.

Choose balls $\hat{B}_{i}$ 's for $\hat{P}$ satisfying $(*)$. Use Lemma 4.2 .3 to find balls $B_{i}$, each with non-empty interior. Clearly these balls satisfy $(*)$ from Lemma 4.2.1 used with $P=\phi \uparrow \hat{P}$. Therefore, to finish the proof, it is enough to show that its property (b) is satisfied. 
So, choose a line $\ell$ in $\mathbb{R}^{n}$ and, by way of contradiction, assume that $\ell$ intersects infinitely many balls $B_{i}$. Then, there is a sequence satisfying (ii) from Lemma 4.2.3. But this and convexity of $\phi$ imply that $\ell$ is below the graph of $\phi$. This gives us the desired contradiction, since such a line cannot intersect any ball $B_{i}$, each ball being chosen strictly above the graph of $\phi$.

\subsection{A Sufficient Condition for $\mathbb{R}^{2}$.}

Throughout this section we assume that $n=2$. Our goal is to show, using the machinery developed in the previous section, that $\phi\left\lceil K \in \mathcal{D}_{1,2}\right.$ for every $\mathcal{C}^{2}$ function $\phi: \mathbb{R} \rightarrow \mathbb{R}$ and compact nowhere dense $K \subset \mathbb{R}$.

We start with the following lemma which, in particular, shows that the function constructed in Proposition 4.1.4 (i.e., with an image of a nowhere dense set having non-empty interior), cannot be continuously differentiable.

Lemma 4.3.1 If $\phi: \mathbb{R} \rightarrow \mathbb{R}$ is $\mathcal{C}^{1}$ and $P$ is compact nowhere dense subset of $\mathbb{R}$, then $\phi[P]$ is also nowhere dense in $\mathbb{R}$.

Proof. Let $Z=\left\{x \in P: \phi^{\prime}(x)=0\right\}$. Then, by Sard's theorem, $\phi[Z]$ has measure zero. Since $Z$ is compact, as $\phi^{\prime}$ is continuous, $\phi[Z]$ is also compact. Therefore, $\phi[Z]$ is nowhere dense in $\mathbb{R}$.

Next, let $J_{k}$ be the closures the component intervals of the complement of $Z$. Since $\phi\left\lceil J_{k}\right.$ is a homeomorphism, each set $M_{k}=\phi\left[P \cap J_{k}\right]$ is nowhere dense, as a homeomorphic image of a nowhere dense set. So, $\phi[P]$ is meager, being equal to a meager set $Z \cup \bigcup_{k} M_{k}$. Being compact, it must be nowhere dense. 
Lemma 4.3.2 Let $\phi: \mathbb{R} \rightarrow \mathbb{R}$ be $\mathcal{C}^{2}$ and $P$ be compact nowhere dense subset of $\mathbb{R}$. For every $x \in \mathbb{R}$ let $\ell_{x}$ be the line tangent to $\phi$ at $\langle x, \phi(x)\rangle$. Then the set $K_{P}=\bigcup_{x \in P} \ell_{x}$ is nowhere dense in the plane.

Proof. First, notice that $K_{P}$ is closed in $\mathbb{R}^{2}$. Indeed, let $\langle a, b\rangle$ be in the closure of $K_{P}$. Choose points $\left\langle a_{k}, b_{k}\right\rangle \in K_{P}$ converging to $\langle a, b\rangle$. For every $k<\omega$ choose $x_{k} \in P$ for which $\left\langle a_{k}, b_{k}\right\rangle \in \ell_{x_{k}}$. By compactness of $P$, choosing a subsequence, if necessary, we can assume that points $x_{k}$ converge to an $\hat{x} \in P$. Since $\phi^{\prime}\left(x_{k}\right)$ converge to $\phi^{\prime}(\hat{x})$, it is easy to see that $\langle a, b\rangle=$ $\left\langle a, \ell_{\hat{x}}(a)\right\rangle \in \ell_{\hat{x}} \subset K_{P}$. So, indeed $K_{P}$ is closed in $\mathbb{R}^{2}$.

Next, we prove that for every vertical line $\ell$, the intersection $K_{P} \cap \ell$ is nowhere dense in $\ell$. By the Kuratowski-Ulam theorem, this implies that $K_{P}$ is meager, so nowhere dense.

So, let $\ell=\{a\} \times \mathbb{R}$. Then $\ell \cap \ell_{x}=\left\langle a, \phi(x)+(a-x) \phi^{\prime}(x)\right\rangle$. In particular, $\ell \cap K_{P}=\{a\} \times \hat{\phi}[P]$, where $\hat{\phi}: \mathbb{R} \rightarrow \mathbb{R}$ is defined as $\hat{\phi}(x)=\phi(x)+(a-x) \phi^{\prime}(x)$. Since $\hat{\phi}$ is $\mathcal{C}^{1}$, as $\phi$ is $\mathcal{C}^{2}$, Lemma 4.3 .1 implied that $\hat{\phi}[P]$ is nowhere dense in $\mathbb{R}$. So, indeed $K_{P}$ is nowhere dense in $\mathbb{R}^{2}$.

Proof of Theorem 4.1.2 For $\mathcal{F}=\mathcal{C}^{2}(\mathbb{R})$. Let $\hat{P} \subset \mathbb{R}$ be compact nowhere dense and $\phi: \mathbb{R} \rightarrow \mathbb{R}$ be $\mathcal{C}^{2}$. We need to show that $\phi \uparrow \hat{P} \in \mathcal{D}_{1,2}$. We proceed as in the previous section.

Choose balls (intervals) $\hat{B}_{i}$ 's for $\hat{P}$ satisfying $(*)$. Use Lemma 4.2 .3 to find balls $B_{i}$, each with non-empty interior. Since, by Lemma 4.3.2, the set $K_{\hat{P}}$ is nowhere dense in $\mathbb{R}^{2}$, we can additionally assume (this is the key trick) that the balls $B_{i}$ are disjoint with $K_{\hat{P}}$. Once again, the balls $B_{i}$ satisfy (*) from Lemma 4.2.1 used with $P=\phi \uparrow \hat{P}$. 
To finish the proof, it is enough to show that the property (b) from Lemma 4.2.1 is satisfied. For this, choose a line $\ell$ in $\mathbb{R}^{2}$ and, by way of contradiction, assume that $\ell$ intersects infinitely many balls $B_{i}$. Then, there is a sequence satisfying (ii) from Lemma 4.2.3. This means, that $\ell$ is equal to the tangent line $\ell_{b} \subset K_{\hat{P}}$. So, $\ell$ could not have intersected any balls $B_{i}$, since all these balls are disjoint with $K_{\hat{P}}$.

\subsection{Discussion}

We note that our results in this chapter have fallen just short of a full characterization of which sets are in $\mathcal{D}_{1, n}$; however, we note that we give in the next chapter a theorem which gives a characterization of the subsets of $\mathbb{R}^{2}$ that are in $\mathcal{D}_{1,2}$. The characterization does not appear to generalize to the sets $\mathcal{D}_{1, n}$ for $n>2$. 


\section{Chapter 5}

\section{Characterizations of Discontinuity Sets}

\subsection{Introduction}

This chapter addresses those functions whose restrictions to general affine sets are continuous. The affine sets in question are those in the families $\mathcal{F}_{k}$ and $\mathcal{F}_{k}^{+}$for various values of $k$. Recall that $\mathcal{F}_{k}^{+}$refers to the family of $k$-flats spanned by $k$ vectors parallel to $k$ axes and that $\mathcal{F}_{k}$ refers to the entire family of $k$-flats. Although there has been some previous mention of $\mathcal{F}_{k}^{+}$-continuous functions in the literature prior to this, the $\mathcal{F}_{k}$ continuous functions have been the object of very little study until now. Our presentation of these results follows our paper [11] very closely. We mention that the results for the $\mathcal{F}_{k}^{+}$-continuous functions are analogous to those for separately continuous functions, while the results for $\mathcal{F}_{k}$-continuous functions seem to be analogous to those for linearly continuous functions, however, they appear 
to be more forthcoming only for fairly large values of $k$. In this notation, the class of $\mathcal{F}_{1}^{+}$-continuous $\left(\mathcal{F}_{1}\right.$-continuous) $f: \mathbb{R}^{n} \rightarrow \mathbb{R}$ is identical with the class of separately (linearly, respectively) continuous functions from $\mathbb{R}^{n}$ to $\mathbb{R}$. Clearly, $\mathcal{F}_{n}=\mathcal{F}_{n}^{+}=\left\{\mathbb{R}^{n}\right\}$, so $\mathcal{F}_{n}^{+}$-continuity is the standard continuity, while $\mathcal{F}_{0}^{+}=\mathcal{F}_{0}$ is the class of all singletons, so that every function $f: \mathbb{R}^{n} \rightarrow \mathbb{R}$ is $\mathcal{F}_{0}$-continuous. So, we concentrate on the cases when $0<k<n$. From Propositions 2.2.7 and 2.2.11 we see that the following relationships hold among these continuities.

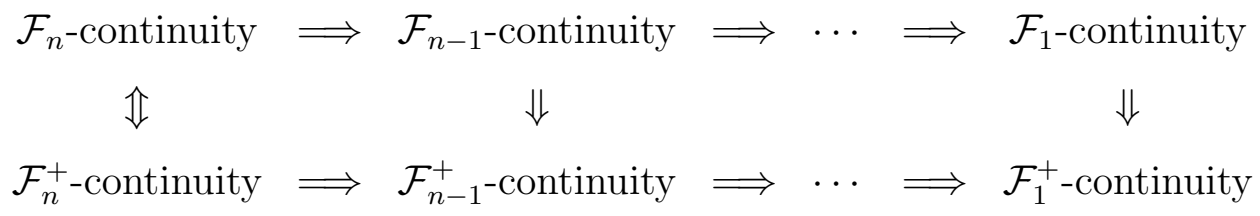

That none of these implications can be reversed follows from Corollary 5.3.5. This corollary will follow from our two primary results on the structure of discontinuity sets of the $\mathcal{F}_{k}$-continuous and $\mathcal{F}_{k}^{+}$-continuous functions.

The $\mathcal{F}_{k}^{+}$-continuous functions on $\mathbb{R}^{n}$ are fairly well documented in the literature. They have been studied in connection with the theory of Sobolev spaces, see e.g. [4]. It is perhaps instructive to think of $\mathcal{F}_{k}^{+}$-continuous functions as those which are continuous when looked at in any $k$ variables separately.

In this chapter, we accomplish three goals:

1. We completely characterize the sets $\mathcal{D}_{k, n}^{+}$. 
2. We derive several structural results on $\mathcal{D}_{k, n}$.

3. We characterize the sets $\mathcal{D}_{k, n}$ when $k \geq \frac{n}{2}$.

\subsection{Characterization of the Sets $\mathcal{D}_{k, n}^{+}$}

Our primary result on the discontinuities of the $\mathcal{F}_{k}^{+}$-continuous functions is the following.

Theorem 5.2.1 $A$ set $D \subset \mathbb{R}^{n}$ is in $\mathcal{D}_{k, n}^{+}$if, and only if, $D$ is an $F_{\sigma^{-} \text {set }}$ whose orthogonal projection on any right $(n-k)$-flat is of first category.

The proof of Theorem 5.2.1 will be presented later in Section 5.5. Below, we discuss some of its consequences.

Proposition 5.2.2 For any $0<k \leq n$, there exists a $D \in \mathcal{D}_{k-1, n}^{+} \backslash \mathcal{D}_{k, n}^{+}$of positive n-dimensional Lebesgue measure.

Proof. Let $K \subset \mathbb{R}$ be a compact, first category set with positive Lebesgue measure (i.e., a "fat" Cantor set). Let $D=K^{k} \times \mathbb{R}^{n-k}$ and note that $D$ has positive measure in $\mathbb{R}^{n}$. Moreover, by Theorem 5.2.1, $D \in \mathcal{D}_{k-1, n}^{+}$, since for any $F \in \mathcal{F}_{n-k+1}$ the projection $\pi_{F}[D]$ is of the form $K^{j} \times \mathbb{R}^{n-k+1-j}$ for some $j>0$, so that $\pi_{F}[D]$ is of first category in $F$. Finally, once again by Theorem 5.2.1, $D \notin \mathcal{D}_{k, n}^{+}$since for $F=\{0\}^{k} \times \mathbb{R}^{n-k}, \pi_{F}[D]=F$ is not of first category in $F$.

We can also derive the following well-known result about the sets of discontinuity for $\mathcal{F}_{k}$-continuous functions. 
Corollary 5.2.3 For any $D \in \mathcal{D}_{k, n}$ and any $F \in \mathcal{F}_{n-k}$ the projection $\pi_{F}[D]$ of first category in $F$.

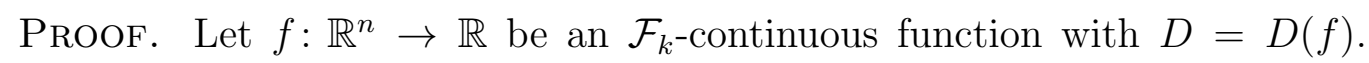
Choose a perpendicular coordinate system for $\mathbb{R}^{n}$ such that $k$-many of the axes are perpendicular to $F$, while the remaining $(n-k)$-many axes are parallel to $F$. Since $f$ is $\mathcal{F}_{k}$-continuous, $f$ is also $\mathcal{F}_{k}^{+}$-continuous in this coordinate system. Hence, by Theorem 5.2.1, $\pi_{F}[D]$ is of first category in $F$.

\section{$5.3 \mathcal{F}_{k}$-continuous Functions and Some Struc- ture of the Sets in $\mathcal{D}_{k, n}$}

Although the $\mathcal{F}_{k}$-continuous functions are a natural refinement of linear continuity, this paper marks their first appearance in the literature. Therefore, we start here with several examples of such functions, the first of which constitutes a generalization of the example (1.2).

Example 5.3.1 For every $n \geq 2$, the following function $f_{n}: \mathbb{R}^{n} \rightarrow \mathbb{R}$, constructed by the first author in [8], is $\mathcal{F}_{n-1}$-continuous and discontinuous precisely at the origin

$f_{n}\left(x_{0}, x_{1}, \ldots, x_{n-1}\right)=\left\{\begin{array}{cl}\frac{x_{0} \prod_{i=0}^{n-1}\left(x_{i}\right)^{2 i}}{\sum_{n=0}^{n-1}\left(x_{i}\right)^{2^{2+i}}} & \text { if }\left\langle x_{0}, x_{1}, \ldots, x_{n-1}\right\rangle \neq\langle 0,0, \ldots, 0\rangle, \\ 0 & \text { if }\left\langle x_{0}, x_{1}, \ldots, x_{n-1}\right\rangle=\langle 0,0, \ldots, 0\rangle .\end{array}\right.$ 
In particular, $f_{3}: \mathbb{R}^{3} \rightarrow \mathbb{R}$ is defined as

$$
f_{3}(x, y, z)=\left\{\begin{array}{cl}
\frac{x^{2} y^{4} z^{16}}{x^{8}+y^{16}+z^{32}} & \text { if }\langle x, y, z\rangle \neq\langle 0,0,0\rangle \\
0 & \text { if }\langle x, y, z\rangle=\langle 0,0,0\rangle
\end{array}\right.
$$

Note that if we define the path $p(t)=\left\langle t^{2^{n}}, t^{2^{n-1}}, \ldots, t^{2^{2}}, t^{2^{1}}\right\rangle$, then along this path $f_{n}(p(t))=\frac{1}{n}$ for $t \neq 0$, while $f_{n}(p(0))=0$.

A more general example of this kind is given by the following result.

Proposition 5.3.2 For every $k<n$ and any compact nowhere dense $K \subset$ $\mathbb{R}$, the set $\{0\}^{k} \times K \times \mathbb{R}^{n-k-1}$ belongs to $\mathcal{D}_{k, n}$. In particular, $\mathcal{D}_{k, n}$ contains the sets of positive $k$-Hausdorff measure. ${ }^{1}$

Proof. For $k=0$ the statement is clearly true, since any function from $\mathbb{R}^{n}$ to $\mathbb{R}$ is $\mathcal{F}_{0}$-continuous. First, we prove, by induction on $n=1,2,3, \ldots$, that

$\left(I_{n}\right)$ the statement is true for $k=n-1$.

For $n=1$ this is true, since then $k=n-1=0$. So, assume that $\left(I_{n}\right)$ holds for some $n$. We need to show $\left(I_{n+1}\right)$.

By $\left(I_{n}\right)$, there exists an $\mathcal{F}_{n-1}$-continuous $g: \mathbb{R}^{n} \rightarrow \mathbb{R}$ with $D(g)=\{\theta\}$, where $\theta=\langle 0,0, \ldots, 0\rangle$. We can assume that $g(\theta)=0$. So, there is a sequence $s=\left\langle s_{m} \in \mathbb{R}^{n}: m<\omega\right\rangle$ converging to $\theta$ such that $\lim _{m \rightarrow \infty}\left|g\left(s_{m}\right)\right|>0$. In particular, there exists a $c>0$ such that $\left|g\left(s_{m}\right)\right|>c$ for every $m<\omega$. Let $S=\left\{s_{m} \in \mathbb{R}^{n}: m<\omega\right\}$. Choose distinct points $y_{j} \in \mathbb{R} \backslash K, j<\omega$, such that

\footnotetext{
${ }^{1}$ For $k=0$ and $k=n-1$, the set $\{0\}^{k} \times K \times \mathbb{R}^{n-k-1}$ is interpreted as $K \times \mathbb{R}^{n-1}$ and $\{0\}^{n-1} \times K$, respectively.
} 
$K$ is the set of accumulation points of $\left\{y_{j}: j<\omega\right\}$. Choose distinct points $x_{j} \in S, j<\omega$, such that

$$
\frac{\left\|x_{j}\right\|}{\operatorname{dist}\left(y_{j}, K\right)}<2^{-j} \text { for every } j<\omega \text {. }
$$

Choose numbers $\varepsilon_{j} \in\left(0,2^{-j}\right)$ such that the sets $B_{j}=B\left(\left\langle x_{j}, y_{j}\right\rangle, \varepsilon_{j}\right)$ are pairwise disjoint and that

$$
0<\frac{\left\|a_{j}\right\|}{\operatorname{dist}\left(b_{j}, K\right)}<2^{-j} \text { for every }\left\langle a_{j}, b_{j}\right\rangle \in \mathbb{R}^{n} \times \mathbb{R} \text { from } B_{j} .
$$

Choose continuous maps $f_{j}: \mathbb{R}^{n-1} \times \mathbb{R} \rightarrow[0,1], j<\omega$, such that $\operatorname{supp}\left(f_{j}\right) \subset$ $B_{j}$ and $f_{j}\left(x_{j}, y_{j}\right)=1$. Define $\hat{g}: \mathbb{R}^{n-1} \times \mathbb{R} \rightarrow \mathbb{R}$ via formula $\hat{g}(x, y)=g(x)$, put $f=\hat{g} \cdot \sum_{j<\omega} f_{j}$, and notice that $f$ is the desired function.

Indeed, $f$ is discontinuous on $\{\theta\} \times K$, since $\{\theta\} \times K$ is in the closure of $T=\left\{\left\langle x_{j}, y_{j}\right\rangle: j<\omega\right\}, f[T] \cap(-c, c)=\emptyset$, and $f[\{\theta\} \times K]=\{0\}$. Moreover, $f$ is continuous at every $p \in \mathbb{R}^{n} \backslash(\{\theta\} \times K)$, since every function $\hat{g} \cdot f_{j}$ is continuous and $p$ has a neighborhood intersecting only finitely many sets $B_{j} \supset \operatorname{supp}\left(g \cdot f_{j}\right)$. Therefore, $D(f)=\{\theta\} \times K$, as required.

To see that $f$ is $\mathcal{F}_{n}$-continuous, choose an $F \in \mathcal{F}_{n}$. If $F$ intersects only finitely many sets $B_{j}$, then clearly $f \uparrow F$ is continuous. On the other hand, if $F$ intersects infinitely many sets $B_{j}$, then, by (5.1), $F$ intersects $\{\theta\} \times K$ and contains lines forming arbitrary small angles with the line $\{\theta\} \times \mathbb{R}$. So, $\{\theta\} \times \mathbb{R} \subset F$, that is, $F=F^{\prime} \times \mathbb{R}$ for some $(n-1)$-flat $F^{\prime} \subset \mathbb{R}^{n}$. Clearly $f\lceil F$ is continuous at all points not in $\{\theta\} \times \mathbb{R}$. It is also continuous at any $p \in\{\theta\} \times \mathbb{R}$, since for any sequence $\left\langle\left\langle c_{j}, d_{j}\right\rangle \in F^{\prime} \times \mathbb{R}: j<\omega\right\rangle$ converging to $p$, we have $\left|f\left(c_{j}, d_{j}\right)\right| \leq\left|g\left(c_{j}\right)\right|$ and $\lim _{j \rightarrow \infty}\left|g\left(c_{j}\right)\right|=|g(\theta)|=0=f(p)$, as 
$g \uparrow F^{\prime}$ is continuous. Therefore, $f$ is indeed $\mathcal{F}_{n}$-continuous, completing the proof.

To finish the proof of the proposition, assume that $0<k<n-1$. By $\left(I_{k+1}\right)$, there exists an $\mathcal{F}_{k^{-}}$continuous $h: \mathbb{R}^{k} \times \mathbb{R} \rightarrow \mathbb{R}$ with $D(h)=\{0\}^{k} \times K$. Define $f: \mathbb{R}^{n} \rightarrow \mathbb{R}$ as $f\left(x_{0}, \ldots, x_{n-1}\right)=h\left(x_{0}, \ldots, x_{k}\right)$. Clearly such an $f$ is $\mathcal{F}_{k}$-continuous and $D(f)=D(h) \times \mathbb{R}^{n-k-1}=\{0\}^{k} \times K \times \mathbb{R}^{n-k-1}$, as required.

The next theorem, on the structure of sets in $\mathcal{D}_{k, n}$, is a natural generalization of Slobodnik's result, Theorem 1.3.7. In its statement, we will use the following terminology.

Let $\mathcal{V}$ be a family of all vector subspaces of $\mathbb{R}^{n}$ and let $\mathcal{V}_{k}=\mathcal{V} \cap \mathcal{F}_{k}$. For a $V \in \mathcal{V}$ let $V^{\perp} \in \mathcal{V}$ denote the perpendicular complement of $V$ and notice that every $x \in \mathbb{R}^{n}$ has a unique representation as $x=v+w$, where $\langle v, w\rangle \in V \times V^{\perp}$. In what follows, we will identify $\langle v, w\rangle \in V \times V^{\perp}$ with $x=v+w$.

Theorem 5.3.3 For every $0<k<n$ and $D \in \mathcal{D}_{k, n}$ there exists a sequence $\left\langle f_{i}\right\rangle_{i<\omega}$ of Lipschitz functions $f_{i}$ from $V_{i} \in \mathcal{V}_{n-k}$ into $V_{i}^{\perp} \in \mathcal{V}_{k}$ whose graphs cover $D$.

Theorem 5.3.3 will be proved in Section 5.6. Below, we discuss some of its consequences.

Recall that Lipschitz maps cannot raise Hausdorff dimension, see e.g. [20]. In particular, Theorem 5.3.3 and Corollary 5.2.3 immediately imply

Corollary 5.3.4 Every $D \in \mathcal{D}_{k, n}$ has Hausdorff dimension $\leq n-k$. Moreover, for every $f_{i}$ from Theorem 5.3.3, the domain of $D \cap f_{i}$ is of first category 
in $V_{i}$.

This stay in contrast with the families $\mathcal{D}_{k, n}^{+}$, which contain the sets of Hausdorff dimension $n$, as shown in Proposition 5.2.2. Notice also that, by Proposition 5.3.2, the upper bound $n-k$ of the Hausdorff dimension of sets in $\mathcal{D}_{k, n}$ is achieved.

Corollary 5.3.5 For $n \geq 2, \mathcal{D}_{0, n}$ is the family of all $F_{\sigma}$-subsets of $\mathbb{R}^{n}$ and

$$
\begin{aligned}
& \{\emptyset\}=\mathcal{D}_{n, n} \subset \mathcal{D}_{n-1, n} \subset \cdots \quad \cdots \quad \mathcal{D}_{1, n} \subset \mathcal{D}_{0, n} \\
& \text { \| } \cap \cap \quad \| \\
& \mathcal{D}_{n, n}^{+} \subset \mathcal{D}_{n-1, n}^{+} \quad \subset \quad \cdots \quad \subset \quad \mathcal{D}_{1, n}^{+} \subset \mathcal{D}_{0, n}^{+}
\end{aligned}
$$

Moreover, all indicated inclusions are proper.

Proof. The inclusions follow from Proposition 2.2.12. The lower row inclusions are strict by Proposition 5.2.2. The upper row inclusions are strict, since, for every $k<n$, the family $\mathcal{D}_{k, n}$ contains (by Proposition 5.3.2) a set of Hausdorff dimension $n-k$, while (by Corollary 5.3.4) $\mathcal{D}_{k+1, n}$ does not contain such a set. This Hausdorff dimension argument also shows that all indicated inclusions between rows are strict.

\subsection{A Characterization of $D_{k, n}$ when $k \geq \frac{n}{2}$.}

Our final main result requires the following notions.

Definition 5.4.1 The topology on $\mathcal{F}_{k}$ is generated by a subbase formed by the sets $\mathcal{F}(U)=\left\{F \in \mathcal{F}_{k}: F \cap U \neq \emptyset\right\}$, where $U$ is an open set in $\mathbb{R}^{n}$. We denote the intersection $\mathcal{F}\left(U_{1}\right) \cap \mathcal{F}\left(U_{2}\right) \cap \cdots \cap \mathcal{F}\left(U_{j}\right)$ as $\mathcal{F}\left(U_{1}, U_{2}, \ldots, U_{j}\right)$. 
Definition 5.4.2 We define $\mathcal{J}_{k, n}$ as the family of all bounded sets $S \subset \mathbb{R}^{n}$ for which there is an increasing sequence $\left\langle\mathcal{L}_{i}: i<\omega\right\rangle$ of closed subsets of $\mathcal{F}_{k}$ such that $\bigcup_{i<\omega} \mathcal{L}_{i}=\mathcal{F}_{k}$ and, for every $i<\omega, S$ is disjoint with the interior $\operatorname{int}\left(\bigcup \mathcal{L}_{i}\right)$ of the set $\bigcup \mathcal{L}_{i} \subset \mathbb{R}^{n}$.

Theorem 5.4.3 Let $0<k<n$ be such that $k \geq \frac{n}{2}$. A set $D \subset \mathbb{R}^{n}$ is in $\mathcal{D}_{k, n}$ if, and only if, $D$ is a countable union of compact sets from $\mathcal{J}_{k, n}$.

Note the theorem provides a characterization of a family $\mathcal{D}_{1,2}$. In particular, Theorem 5.4.3 provides a solution Kronrod's problem.

\subsection{Proof of Theorem 5.2.1}

We will use the following two lemmas. The first, is just a convenient presentation of well know result. It will be used in what follows for $Z=\mathbb{R}^{k}$.

Lemma 5.5.1 Let $Z$ be locally compact and $\sigma$-compact, and assume that $f: Z \times \mathbb{R}^{m} \rightarrow \mathbb{R}$ is separately continuous as a function of $m+1$ variables. If $K \subset Z \times \mathbb{R}^{m}$ has compact projection $\pi_{Z}[K]$ and there is an $\varepsilon>0$ such that $\operatorname{osc}(f, p) \geq \varepsilon$ for all $p \in K$, then $\pi_{\mathbb{R}^{m}}[K]$ is nowhere dense in $\mathbb{R}^{m}$. In particular, $\pi_{\mathbb{R}^{m}}[D(f)]$ is of first category in $\mathbb{R}^{m}$.

Proof. Let $Y \subset Z$ be compact whose interior contains $\pi_{Z}[K]$ and let $\bar{f}$ be a restriction of $f$ to $Y \times \mathbb{R}^{m}$. Then $K \subset\left\{p \in Y \times \mathbb{R}^{m}: \operatorname{osc}(\bar{f}, p) \geq \varepsilon\right\}$. Also, clearly $\bar{f}(\cdot, x)$ is continuous for every $x \in \mathbb{R}^{m}$ and, for every $y \in Y, \bar{f}(y, \cdot)$ is quasi-continuous, as it is separately continuous on $\mathbb{R}^{m}$, see e.g. [4, theorem 2.4]. Therefore, $\bar{f}$ satisfies the assumptions of [4, corollary 3.8], so the set 
$\pi_{\mathbb{R}^{m}}\left[\left\{p \in Y \times \mathbb{R}^{m}: \operatorname{osc}(\bar{f}, p) \geq \varepsilon\right\}\right]$ is nowhere dense in $\mathbb{R}^{m}$. Thus, so is its subset $\pi_{\mathbb{R}^{m}}[K]$, as required.

Since $D(f)$ is a countable union of the sets $K$ as in the assumptions, $\pi_{\mathbb{R}^{m}}[D(f)]$ is of first category in $\mathbb{R}^{m}$.

The following lemma is a variant of [25, lemma 9].

Lemma 5.5.2 If $D$ is a compact subset of $\mathbb{R}^{n}$ such that its orthogonal projection onto each $F \in \mathcal{F}_{n-k}^{+}$is nowhere dense, then there exists an $\mathcal{F}_{k}^{+}$ continuous function $f: \mathbb{R}^{n} \rightarrow[0,1]$ such that $D(f)=D$ and $\operatorname{osc}(f, z)=1$ for all $z \in D$.

Proof. Let $\mathcal{V}_{n-k}^{+}$be the family of all linear spaces $V \in \mathcal{F}_{n-k}^{+}$and let $\left\{M_{i}: i<\left(\begin{array}{c}n \\ n-k\end{array}\right)\right\}$ be its enumeration. Then, by the assumption, $D$ is contained in a finite union $Z=\bigcup_{V \in \mathcal{V}_{n-k}^{+}} \pi_{V}^{-1}\left(\pi_{V}[D]\right)$, a closed nowhere dense set.

Construct, by induction on $j<\omega$, a sequence $\left\{U_{j} \subset \mathbb{R}^{n} \backslash Z: j<\omega\right\}$ of disjoint non-empty open balls satisfying the conditions (a), (b), and (F) with $\mathcal{S}=\mathcal{F}_{k}^{+}$of Lemma 2.3.1. This can be done by: choosing a countable dense subset $E$ of $D$; fixing a sequence $\left\langle p_{j} \in E: j<\omega\right\rangle$, so that each point of $E$ occurs infinitely many times; for every $j<\omega$, choosing a ball $U_{j} \subset B\left(p_{j}, 2^{-j}\right)$, so that its closure $\operatorname{cl}\left(U_{j}\right)$ is disjoint with $Z \cup \bigcup_{i<j} \bigcup_{V \in \mathcal{V}_{n-k}^{+}} \pi_{V}^{-1}\left(\pi_{V}\left[\operatorname{cl}\left(U_{i}\right)\right]\right)$. The choice of $U_{j}$ is possible, since the construction insures that for every $V \in \mathcal{V}_{n-k}^{+}$the sets $\pi_{V}\left[\operatorname{cl}\left(U_{i}\right)\right], i<j$, are disjoint with $\pi_{V}[D]$.

Choose functions $f_{j}$ as in Lemma 2.3.1. Then $f=\sum_{j<\omega} f_{j}$ is as desired.

Proof of Theorem 5.2.1. Let $f: \mathbb{R}^{n} \rightarrow \mathbb{R}$ be an $\mathcal{F}_{k}^{+}$-continuous function and let $D=D(f)$. Clearly, $D$ must be $F_{\sigma}$, as this is true for any set of points 
of discontinuity of a function $f: \mathbb{R}^{n} \rightarrow \mathbb{R}$, see e.g. [35]. Let $F \in \mathcal{F}_{n-k}^{+}$. We need to show that $\pi_{F}[D]$ is of first category in $F$.

Since a translation of $F$ does not change this property, we can assume that $F \in \mathcal{V}_{n-k}$. So, $F$ is an $(n-k)$-dimensional linear subspace of $\mathbb{R}^{n}$. Let $F^{\perp}$ be a $k$-dimensional linear subspace of $\mathbb{R}^{n}$ perpendicular to $F$. Identify $\mathbb{R}^{n}$ with $F \times F^{\perp}$. Then $f$ can be treated as an $\mathcal{F}_{k}^{+}$-continuous function on $\mathbb{R}^{n-k} \times \mathbb{R}^{k}$. Then, by Lemma 5.5.1, $\pi[D]$ is of first category in $\mathbb{R}^{n-k}$ and so, $\pi_{F}[D]$ is of first category in $F$.

To prove the converse implication, fix an $F_{\sigma}$ subset $D$ of $\mathbb{R}^{n}$ whose orthogonal projection on any $F \in \mathcal{F}_{n-k}^{+}$is of first category. In particular, there exists a sequence $\left\langle D_{j}: j<\omega\right\rangle$ of compact sets such that $D=\bigcup_{j<\omega} D_{j}$. Now, each $D_{j}$ satisfies the assumptions of Lemma 5.5.2. Therefore, for every $j<\omega$, there exists an $\mathcal{F}_{k}^{+}$-continuous function $f_{j}: \mathbb{R}^{n} \rightarrow[0,1]$ with the property that $D\left(f_{j}\right)=D_{j}=\left\{z \in \mathbb{R}^{n}: \operatorname{osc}\left(f_{j}, z\right)=1\right\}$. Define $f=\sum_{j<\omega} 3^{-j} f_{j}$. Then $f$ is $\mathcal{F}_{k}^{+}$-continuous, as a uniform limit of such functions. Moreover, $f$ is discontinuous precisely on $D$, since for any $z \in D_{i} \backslash \bigcup_{j<i} D_{j}$ we have osc $\left(\sum_{j \leq i} 3^{-j} f_{j}, z\right)=3^{-i}$, while the range of $\sum_{i<j<\omega} 3^{-j} f_{j}$ is contained in $\left[0,3^{-i}\right)$.

\subsection{Proof of Theorem 5.3.3}

The following lemma translates the formulation of Theorem 5.3.3: it implies that it is enough to prove that any $D \in \mathcal{D}_{k, n}$ can be covered by countably many sets $K$ with some nice projection properties. In what follows, $K-K$ refers to the set $\{p-q: p, q \in K\}$. 
Lemma 5.6.1 Assume that $K \subset \mathbb{R}^{n}, V \in \mathcal{V}_{n-k}$, and $C>0$ have the property that $\|z\| \leq C\left\|\pi_{V}(z)\right\|$ for every $z \in K-K$. Then $K$ is contained in a graph of a Lipschitz function from $V$ into $V^{\perp}$.

ProOF. Let $g=\left\{\left\langle\pi_{V}(z), \pi_{V^{\perp}}(z)\right\rangle: z \in K\right\}$ and notice that $g$ is a Lipschitz function from $\pi_{V}[K]$ into $V^{\perp}$, since for every $z_{0}, z_{1} \in K$

$$
\begin{aligned}
\left\|\pi_{V^{\perp}}\left(z_{0}\right)-\pi_{V^{\perp}}\left(z_{1}\right)\right\|^{2} & =\left\|\pi_{V^{\perp}}\left(z_{0}-z_{1}\right)\right\|^{2} \\
& =\left\|z_{0}-z_{1}\right\|^{2}-\left\|\pi_{V}\left(z_{0}-z_{1}\right)\right\|^{2} \\
& \leq C^{2}\left\|\pi_{V}\left(z_{0}-z_{1}\right)\right\|^{2}-\left\|\pi_{V}\left(z_{0}-z_{1}\right)\right\|^{2} \\
& =\left(C^{2}-1\right)\left\|\pi_{V}\left(z_{0}-z_{1}\right)\right\|^{2} \\
& =\left(C^{2}-1\right)\left\|\pi_{V}\left(z_{0}\right)-\pi_{V}\left(z_{1}\right)\right\|^{2} .
\end{aligned}
$$

Since every partial Lipschitz function from $\mathbb{R}^{k}$ into $\mathbb{R}^{m}$ can be extended to an entire Lipschitz function (see e.g. [19, p. 80]), the result follows.

The following lemma is a generalization of [45, lemma 2].

Lemma 5.6.2 For every $0<k<n, D \in \mathcal{D}_{k, n}$, and $V \in \mathcal{V}_{n-k+1}$, there exists a countable partition $\mathcal{K}$ of $D$ with the following property.

$(\dagger)$ For every $K \in \mathcal{K}$ there exist a $c_{K}>0$ and a perpendicular decomposition $\left\langle L_{K}, W_{K}\right\rangle \in \mathcal{V}_{1} \times \mathcal{V}_{n-k}$ of $V$ such that $\left\|\pi_{L_{K}}(z)\right\| \leq c_{K}\left\|\pi_{W_{K}}(z)\right\|$ for every $z \in K-K$.

Proof. Let $f: \mathbb{R}^{n} \rightarrow \mathbb{R}$ be an $\mathcal{F}_{k}$-continuous function with $D(f)=D$. Then, there exists a countable cover $\mathcal{P}$ of $D$ by the compact sets such that for every $P \in \mathcal{P}$ there is an $\varepsilon>0$ for which $\operatorname{osc}(f, z) \geq \varepsilon$ for every $z \in P$. It 
is enough to show that every $P \in \mathcal{P}$ admits a countable $\mathcal{K}_{P}$ of $P$ satisfying property $(\dagger)$. So, fix a $P \in \mathcal{P}$ and an associated $\varepsilon$.

Choose an arbitrary perpendicular decomposition $\langle L, W\rangle \in \mathcal{V}_{1} \times \mathcal{V}_{n-k}$ of $V$, fix a non-zero $u \in L$, and for $v \in L$ let $a_{v} \in \mathbb{R}$ be such that $v=a_{v} u$. Define function $g:\left(V^{\perp} \times L\right) \times W \rightarrow \mathbb{R}^{n}$ via $g(\langle y, v\rangle, w)=y+v+a_{v} w=$ $y+a_{v}(u+w)$. Of course, the restriction $\bar{g}$ of $g$ to $V^{\perp} \times(L \backslash\{0\}) \times W$ establishes a homeomorphism between this set and $\mathbb{R}^{n} \backslash L^{\perp}$.

For every $p \in \mathbb{R}^{n}$, let $f_{p}:\left(V^{\perp} \times L\right) \times W \rightarrow \mathbb{R}$ be defined as $f(p+g(d))$ for $d$ in the domain. Notice that $f_{p}(\cdot, w)$ is continuous, since $p+g\left[\left(V^{\perp} \times L\right) \times\{w\}\right]$ is a $k$-flat, a $p$-translation of a vector space spanned by $V^{\perp}$ and a vector $u+w \in V$. Moreover, $f_{p}(\langle y, v\rangle, \cdot)$ is separately continuous, upon identification of $W$ with $\mathbb{R}^{n-k}$.

Let us define $S_{p}=\left\{d: \pi_{L}(g(d)) \neq 0 \& p+g(d) \in P\right\}$. Then, $\operatorname{osc}\left(f_{p}, d\right)=$ $\operatorname{osc}(f, p+g(d)) \geq \varepsilon$ for every $d \in S_{p}$, since such $d$ belongs to the domain of the homeomorphism $\bar{g}$. Moreover, it is easy to see that $\pi_{V^{\perp} \times L}\left[S_{p}\right] \subset \pi_{V^{\perp} \times L}[P]$ is bounded, so its closure is compact. Thus, by Lemma 5.5.1, there exists a dense open set $U_{p} \subset W$ such that $\pi_{W}\left[S_{p}\right] \cap U_{p}=\emptyset$.

Now, consider a countable basis $\mathcal{B}$ of $W$ formed by the open balls $B(w, c)$, $c>0$. For every such ball let $P_{w, c}=\left\{p \in P: B(w, c) \subset U_{p}\right\}$. We claim that $K=P_{w, c}$ satisfies $(\dagger)$ with $c_{K}=\frac{\|u+w\|}{c}, L_{K}$ spanned by $u+w$, and $W_{K}$ being the perpendicular complement of $L_{K}$ in $W$.

Indeed, let $z=q-p$, where $p, q \in P_{w, c}$. If $\pi_{L}(z)=0$, then clearly the inequality holds. So, assume that $\pi_{L}(z) \neq 0$. Then, $z=g(d)$ for some $d \in S_{p}$. In particular, $\pi_{W}(d) \notin B(w, c)$, that is, $\left\|\pi_{W}(d)-w\right\| \geq c$. 
Now, $z=g(d)=\pi_{V^{\perp}}(d)+a_{\pi_{L}(d)} u+a_{\pi_{L}(d)} \pi_{W}(d)$. Therefore,

$$
\pi_{L_{K}}(z)=a_{\pi_{L}(d)}(u+w) \quad \& \quad \pi_{W_{K}}(z)=a_{\pi_{L}(d)}\left(\pi_{W}(d)-w\right)
$$

as $\pi_{W_{K}}(z)=\pi_{W}(z)-\pi_{L_{K}}(z)=\left(a_{\pi_{L}(d)} u+a_{\pi_{L}(d)} \pi_{W}(d)\right)-a_{\pi_{L}(d)}(u+w)$. So,

$$
\begin{aligned}
\left\|\pi_{L_{K}}(z)\right\| & =\left|a_{\pi_{L}(d)}\right|\|u+w\| \\
& \leq\left|a_{\pi_{L}(d)}\right|\|u+w\| \frac{\left\|\pi_{W}(d)-w\right\|}{c} \\
& =\frac{\|u+w\|}{c}\left\|\pi_{W_{K}}(z)\right\|,
\end{aligned}
$$

as required.

Proof of Theorem 5.3.3. Let $D \in \mathcal{D}_{k, n}$. We will prove the following property by induction on $\ell \leq k$ :

$\left(I_{\ell}\right)$ There exists a countable partition $\mathcal{P}_{\ell}$ of $D$ such that for every $P \in \mathcal{P}_{\ell}$ there exist $V_{P} \in \mathcal{V}_{n-\ell}$ and $C_{P}>0$ such that $\|z\| \leq C_{P}\left\|\pi_{V_{P}}(z)\right\|$ for any $z \in P-P$.

For $\ell=0$ the property $\left(I_{\ell}\right)$ is satisfied with $\mathcal{P}=\{D\}, V_{D}=\mathbb{R}^{n}$, and $C_{D}=1$. So, assume that for some $\ell<k$ the property $\left(I_{\ell}\right)$ holds. We need to show $\left(I_{\ell+1}\right)$.

So, fix a $P \in \mathcal{P}_{\ell}$ and let $V \in \mathcal{V}_{n-k+1}$ be contained in $V_{P}$. By Lemma 5.6.2, there exists a partition $\mathcal{K}_{P}$ of $D$ such that for every $K \in \mathcal{K}_{P}$ there exist a $c_{K}^{P}>0$ and a perpendicular decomposition $\left\langle L_{K}^{P}, W_{K}^{P}\right\rangle \in \mathcal{V}_{1} \times \mathcal{V}_{n-k}$ of $V$ such that $\left\|\pi_{L_{K}^{P}}(z)\right\| \leq c_{K}^{P}\left\|\pi_{W_{K}^{P}}(z)\right\|$ for every $z \in K-K$.

Then the partition $\mathcal{P}_{\ell+1}=\left\{P \cap K: P \in \mathcal{P}_{\ell} \& K \in \mathcal{K}_{P}\right\}$ satisfies $\left(I_{\ell+1}\right)$, with $V_{K}^{P} \in \mathcal{V}_{n-\ell-1}$ being a subspace of $V_{P}$ perpendicular to $L_{K}^{P}$. Indeed, 
for any point $z \in(P \cap K)-(P \cap K) \subset(P-P) \cap(K-K)$ we have the inequalities $\left\|\pi_{L_{K}^{P}}(z)\right\| \leq c_{K}^{P}\left\|\pi_{W_{K}^{P}}(z)\right\| \leq c_{K}^{P}\left\|\pi_{V_{K}^{P}}(z)\right\|$, as $W_{K}^{P} \subset V_{K}^{P}$. Therefore, $\left\|\pi_{V_{P}}(z)\right\|^{2}=\left\|\pi_{L_{K}^{P}}(z)\right\|^{2}+\left\|\pi_{V_{K}^{P}}(z)\right\|^{2} \leq\left(\left(c_{K}^{P}\right)^{2}+1\right)\left\|\pi_{V_{K}^{P}}(z)\right\|^{2}$. In particular, $\|z\| \leq C_{P}\left\|\pi_{V_{P}}(z)\right\| \leq C_{P} \sqrt{\left(c_{K}^{P}\right)^{2}+1}\left\|\pi_{V_{K}^{P}}(z)\right\|$. In other words, $\left(I_{\ell+1}\right)$ is satisfied with $C_{K}^{P}=C_{P} \sqrt{\left(c_{K}^{P}\right)^{2}+1}$. This finishes the inductive proof of $\left(I_{\ell}\right)^{\prime}$ 's.

The theorem is concluded by noticing that the partition given by $\left(I_{k}\right)$ is as required, as implied by Lemma 5.6.1.

\subsection{Proof of Theorem 5.4.3}

Recall, that the topology on $\mathcal{F}_{k}$ is defined by a subbasis formed by the sets $\mathcal{F}(U)=\left\{F \in \mathcal{F}_{k}: F \cap U \neq \emptyset\right\}$, where $U$ is an open subset of $\mathbb{R}^{n}$, and that $\mathcal{F}\left(U_{0}, U_{1}, \ldots, U_{j}\right)$ is defined as $\bigcap_{i \leq j} \mathcal{F}\left(U_{i}\right)$. Our proof will require few facts about this topology on $\mathcal{F}_{k}$.

Fact 5.7.1 If $F \in \mathcal{F}_{k}$ and the points $x_{0}, x_{1}, \ldots, x_{k} \in F$ are in general position, then the sets

$$
\left\{\mathcal{F}\left(B\left(x_{0}, r\right), B\left(x_{1}, r\right), \ldots, B\left(x_{k}, r\right)\right): r>0\right\}
$$

form a basis at $F$.

Proof. For $r>0$ let $\mathcal{F}(r) \stackrel{\text { def }}{=} \mathcal{F}\left(B\left(x_{0}, r\right), B\left(x_{1}, r\right), \ldots, B\left(x_{k}, r\right)\right.$ and notice that if $r_{1}<r_{2}$, then $\mathcal{F}\left(r_{1}\right) \subset \mathcal{F}\left(r_{2}\right)$.

The result follows from the following property: 
(A) For every open subset $U \subset \mathbb{R}^{n}$, if $U \cap F \neq \emptyset$, then there exists an $r>0$ such that $\mathcal{F}(r) \subset \mathcal{F}(U)$.

Indeed, any open set containing $F$ contains a subset of the form $\bigcap_{i=1}^{j} \mathcal{F}\left(U_{i}\right)$, where each $U_{i} \subset \mathbb{R}^{n}$ is an open and intersects $F$. By (A), for every $i \leq j$ there exists an $r_{i}>0$ such that $F \in \mathcal{F}\left(r_{i}\right) \subset \mathcal{F}\left(U_{i}\right)$. Put $r=\min _{i \leq j} r_{i}$. Then $F \in \mathcal{F}(r) \subset \bigcap_{i \leq j} \mathcal{F}\left(U_{i}\right)$, as required.

We will finish the proof by showing that (A) holds. So, fix an open $U \subset \mathbb{R}^{n}$ with $U \cap F \neq \emptyset$ and let $x \in U \cap F$.

Since all $k$-flats are affine sets, the points of $F$ are precisely those which can be expressed as an affine combination of $(k+1)$-many points in $F$ in general position, see for instance [22] or [28, Section 1.2]. In particular, there exist $\beta_{0}, \beta_{1}, \ldots, \beta_{k} \in \mathbb{R}$ such that $\sum_{i=0}^{k} \beta_{i}=1$ and $x=\sum_{i=1}^{k} \beta_{i} x_{i}$.

Define a function $g:\left(\mathbb{R}^{n}\right)^{k+1} \rightarrow \mathbb{R}^{n}$ by setting

$$
g\left(z_{0}, z_{1}, \ldots, z_{k}\right)=\sum_{i=0}^{k} \beta_{i} z_{i}
$$

Note that $g$ is continuous and that $g\left(x_{0}, x_{1}, \ldots, x_{k}\right)=x \in U \cap F$. So, there is an $r>0$ such that if $\left\langle z_{i}\right\rangle_{i=0}^{k} \in \prod_{i=0}^{k} B\left(x_{i}, r\right)$, then $g\left(z_{0}, z_{1}, \ldots, z_{k}\right) \in U$. It is enough to show that $\mathcal{F}(r) \subset \mathcal{F}(U)$.

Indeed, let $F^{\prime} \in \mathcal{F}(r)$. Then, there exists a $\left\langle z_{i}\right\rangle_{i=0}^{k} \in \prod_{i=0}^{k}\left(F^{\prime} \cap B\left(x_{i}, r\right)\right)$. As $z=g\left(z_{0}, z_{1}, \ldots, z_{k}\right)$ is an affine combination of points $z_{0}, z_{1}, \ldots, z_{n} \in F^{\prime}$, $z$ belongs to $F^{\prime}$. Also, the choice of $r$ insures that $z \in U$. Thus, $z \in F^{\prime} \cap U$ and so, $F^{\prime} \in \mathcal{F}(U)$, as required.

Fact 5.7.2 If $Z$ is a closed subset of $\mathcal{F}_{k}$, then $\bigcup Z$ is a closed subset of $\mathbb{R}^{n}$. 
Proof. Let $\left\langle z_{0}^{i}: i<\omega\right\rangle$ be a sequence of points in $\bigcup Z$ with the property that $\lim _{i \rightarrow \infty} z_{0}^{i}=z_{0} \in \mathbb{R}^{n}$. We will show that $z_{0} \in \bigcup Z$.

For each $i<\omega$ there exists a $k$-flat $F_{i} \in Z$ with $z_{0}^{i} \in F_{i}$. Choose the points $z_{1}^{i}, \ldots, z_{k}^{i} \in F_{i}$ such that $\left\|z_{j}^{i}-z_{\ell}^{i}\right\|=1$ for all $j<\ell \leq k$. Since the sequence $\left\langle z_{0}^{i}, \ldots, z_{k}^{i}\right\rangle_{i<\omega}$ is bounded, choosing subsequence, if necessary, we can ensure that it converges to a point $\left\langle z_{0}, \ldots, z_{k}\right\rangle \in \mathbb{R}^{k+1}$. Clearly we have $\left\|z_{j}-z_{\ell}\right\|=1$ for all $j<\ell \leq k$. In particular, the points $z_{0}, \ldots, z_{k}$ are in general position. Thus, they all belong to some $k$-flat $F$ and, by Fact 5.7.1, the family $\left\{\mathcal{F}\left(B\left(z_{0}, r\right), B\left(z_{1}, r\right), \ldots, B\left(z_{k}, r\right)\right): r>0\right\}$ forms a basis at $F$. Since every set $\mathcal{F}\left(B\left(z_{0}, r\right), B\left(z_{1}, r\right), \ldots, B\left(z_{k}, r\right)\right)$ contains some $F_{i} \in Z, F$ is in the closure of $Z$, that is, $F \in Z$. In particular, $z_{0} \in F \subset \bigcup Z$, as required.

Now that some basic facts about our topology have been laid out, consider the family $\mathcal{J}_{k, n}$ discussed earlier. We prove the following structural result about $\mathcal{J}_{k, n}$ which will be essential to the proof of Theorem 5.4.3.

Fact 5.7.3 If $S \in \mathcal{J}_{k, n}$, then $\operatorname{cl}(S) \in \mathcal{J}_{k, n}$ is nowhere dense.

ProOF. Let $\left\{\mathcal{L}_{i}: i<\omega\right\}$ be an increasing sequence of closed subsets of $\mathcal{F}_{k}$ which justifies that $S$ belongs to $\mathcal{J}_{k, n}$. Notice that

$$
\mathbb{R}^{n}=\bigcup \mathcal{F}_{k}=\bigcup_{i<\omega} \bigcup \mathcal{L}_{i}=\bigcup_{i<\omega} \operatorname{int}\left(\bigcup \mathcal{L}_{i}\right) \cup \bigcup_{i<\omega}\left(\bigcup \mathcal{L}_{i} \backslash \operatorname{int}\left(\bigcup \mathcal{L}_{i}\right)\right) .
$$

Define $G=\bigcup_{i<\omega} \operatorname{int}\left(\bigcup \mathcal{L}_{i}\right)$. Then $G$ is an open in $\mathbb{R}^{n}$, being the union of open sets. Moreover, by Fact 5.7.2, each set $\bigcup \mathcal{L}_{i} \backslash \operatorname{int}\left(\bigcup \mathcal{L}_{i}\right)$ is closed nowhere dense in $\mathbb{R}^{n}$. Since clearly $\mathbb{R}^{n} \backslash G$ is a subset of $\bigcup_{i<\omega}\left(\bigcup \mathcal{L}_{i} \backslash \operatorname{int}\left(\bigcup \mathcal{L}_{i}\right)\right), \mathbb{R}^{n} \backslash G$ 
is of first category in $\mathbb{R}^{n}$. So, being closed, it is nowhere dense. As $\operatorname{cl}(S)$ is disjoint with $G=\bigcup_{i<\omega} \operatorname{int}\left(\bigcup \mathcal{L}_{i}\right)$, it is nowhere dense and belongs to $\mathcal{J}_{k, n}$.

Our characterization of $\mathcal{D}_{k, n}$, for $k \geq \frac{n}{2}$, follows from the next three lemmas.

Lemma 5.7.4 Let $k$ and $n$ be integers, with $n>k \geq \frac{n}{2}$. Let $f: \mathbb{R}^{n} \rightarrow \mathbb{R}$ be $\mathcal{F}_{k}$-continuous and let $\delta, \epsilon>0$ be given. If $B=B[N]$ for some $N \in(0, \infty)$, then the set $Z_{\delta, \epsilon} \stackrel{\text { def }}{=}\left\{F \in \mathcal{F}_{k}: \omega(f \uparrow(F \cap B), \delta) \leq \epsilon\right\}$ is closed in $\mathcal{F}_{k}$.

Proof. Let $\epsilon, \delta>0$ be given and let $Z=Z_{\delta, \epsilon}$. It is enough to show that the complement of $Z, Z^{c}=\mathcal{F}_{k} \backslash Z$, is open. So, fix an $F \in Z^{c}$. We will find an open neighborhood $\mathcal{W}$ of $F$ disjoint with $Z$.

Let $V \in \mathcal{V}_{k}$ be such that $F=x_{0}+V$ for some $x_{0} \in F$ and let $W=V^{\perp}$. Choose the points $x_{0}, x_{1}, \ldots, x_{k} \in F$ in general position. The set $\mathcal{W}$ will be of the form $\mathcal{F}(r)=\mathcal{F}\left(B\left(x_{0}, r\right), B\left(x_{1}, r\right), \ldots, B\left(x_{k}, r\right)\right)$ for some $r>0$. Invoking Lemma ??, we can choose an $r>0$ small enough so that for any $y=\left\langle y_{i}\right\rangle_{i \leq k} \in \prod_{i \leq k} B\left(x_{i}, r\right)$ the points from $Y=\left\{y_{i}: i \leq k\right\}$ are in general position. In particular, there is a unique $F(y) \in \mathcal{F}_{k}$ containing $Y$.

Our proof of the lemma is based on the following claim.

Claim 5.7.5 For every $z \in F$ and $y=\left\langle y_{i}\right\rangle_{i \leq k} \in \prod_{i \leq k} B\left(x_{i}, r\right)$, the intersection $(z+W) \cap F(y)$ contains a unique point $h_{z}(y)$. Moreover, the mapping $h_{z}: \prod_{i \leq k} B\left(x_{i}, r\right) \rightarrow \mathbb{R}^{n}$ is continuous.

First, notice that the claim implies the lemma. To see this, we will show that, decreasing $r$, if necessary, $\mathcal{F}(r) \subset Z^{c}$. So, choose an arbitrary $k$-flat 
from $\mathcal{F}(r)$. It is of the form $F(y)$ for some $y=\left\langle y_{i}\right\rangle_{i \leq k} \in \prod_{i \leq k} B\left(x_{i}, r\right)$. We will show that $F(y) \in Z^{c}$ provided $r$ small enough.

As $F \in Z^{c}$, we have $\omega(f \uparrow(F \cap B), \delta)>\epsilon$. So there are $z_{0}, z_{1} \in F \cap B$ for which $\left\|z_{1}-z_{0}\right\|<\delta$ and $\left|f\left(z_{1}\right)-f\left(z_{0}\right)\right|>\epsilon$. Since $f \nmid F$ is continuous, we can choose $z_{0}, z_{1} \in \operatorname{int}(B)=B_{0}$.

Choose an $\varepsilon_{0}>0$ such that ||$z_{1}-z_{0}||+2 \varepsilon_{0}<\delta$ and $\left|f\left(z_{1}\right)-f\left(z_{0}\right)\right|-2 \varepsilon_{0}>\epsilon$. For $i<2, z_{i}+W$ is contained in a $k$-flat (as it has dimension $n-k \leq k$ ). Therefore, function $f \uparrow\left(z_{i}+W\right)$ is continuous at $z_{i}$ and so, there exists a $\delta_{0} \in\left(0, \varepsilon_{0}\right)$ such that $\left|f\left(z_{i}\right)-f(z)\right|<\varepsilon_{0}$ whenever $z \in\left(z_{i}+W\right) \cap B\left(z_{i}, \delta_{0}\right)$. Since functions $h_{z_{i}}$ are continuous, we can decrease $r$ so that $\left\|h_{z_{i}}(y)-z_{i}\right\|=$ $\left\|h_{z_{i}}(y)-h_{z_{i}}\left(x_{0}, \ldots, x_{k}\right)\right\|<\delta_{0}$ whenever $y \in \prod_{i \leq k} B\left(x_{i}, r\right)$. For $i<2$ define $z_{i}^{\prime} \stackrel{\text { def }}{=} h_{z_{i}}(y) \in F(y)$ and note that

$$
\begin{aligned}
\left\|z_{0}^{\prime}-z_{1}^{\prime}\right\| & =\left\|h_{z_{0}}(y)-h_{z_{1}}(y)\right\| \\
& \leq\left\|h_{z_{0}}(y)-z_{0}\right\|+\left\|z_{0}-z_{1}\right\|+\left\|h_{z_{1}}(y)-z_{1}\right\| \\
& <\delta_{0}+\delta-2 \varepsilon_{0}+\delta_{0}<\delta-2 \varepsilon_{0}+2 \varepsilon_{0}=\delta .
\end{aligned}
$$

Furthermore,

$$
\begin{aligned}
\left|f\left(z_{0}^{\prime}\right)-f\left(z_{1}^{\prime}\right)\right| & =\left|f\left(z_{0}^{\prime}\right)-f\left(z_{0}\right)+f\left(z_{0}\right)-f\left(z_{1}\right)+f\left(z_{1}\right)-f\left(z_{1}^{\prime}\right)\right| \\
& =\left|-\left[f\left(z_{1}\right)-f\left(z_{0}\right)\right]-\left[f\left(z_{0}\right)-f\left(z_{0}^{\prime}\right)\right]-\left[f\left(z_{1}^{\prime}\right)-f\left(z_{1}\right)\right]\right| \\
& \geq\left|f\left(z_{1}\right)-f\left(z_{0}\right)\right|-\left|f\left(z_{0}\right)-f\left(z_{0}^{\prime}\right)\right|-\left|f\left(z_{1}\right)-f\left(z_{1}^{\prime}\right)\right| \\
& >\epsilon+2 \varepsilon_{0}-\varepsilon_{0}-\varepsilon_{0}=\epsilon .
\end{aligned}
$$


The last two computations justify the statement $\omega(f \uparrow(F(y) \cap B), \delta)>\epsilon$. Hence, indeed $F(y) \in Z^{c}$ as required.

The above argument reduces the proof of the lemma to that of Claim 5.7.5. So, we proceed to prove the claim. For this, fix $y$ and $z$ as in the assumptions of Claim 5.7.5. First notice that $\left\langle\pi_{F}\left(y_{i}\right)\right\rangle_{i \leq k} \in \prod_{i \leq k} B\left(x_{i}, r\right)$. Thus, the points $\pi_{F}\left(y_{0}\right), \ldots, \pi_{F}\left(y_{k}\right)$ are in general position. In particular, $z$ has a unique representation as $z=\sum_{i \leq k} \alpha_{i}(y) \pi_{F}\left(y_{i}\right)$, where $\sum_{i \leq k} \alpha_{i}(y)=1$. We shall show that $(z+W) \cap F(y)=\left\{\sum_{i \leq k} \alpha_{i}(y) y_{i}\right\}$, that is, that $h_{z}(y)=\sum_{i \leq k} \alpha_{i}(y) y_{i}$.

Indeed, every $p \in F(y)$ has a unique representation as $p=\sum_{i \leq k} \alpha_{i} y_{i}$ with $\sum_{i \leq k} \alpha_{i}=1$. This $p$ belongs to $z+W$ if, and only if,

$$
\sum_{i \leq k} \alpha_{i}(y) \pi_{F}\left(y_{i}\right)=z=\pi_{F}(p)=\pi_{F}\left(\sum_{i \leq k} \alpha_{i} y_{i}\right)=\sum_{i \leq k} \alpha_{i} \pi_{F}\left(y_{i}\right)
$$

In particular, $p$ belongs to $z+W$ if, and only if, $\alpha_{i}=\alpha_{i}(y)$ for every $i \leq k$ if, and only, if $p=\sum_{i \leq k} \alpha_{i}(y) y_{i}$. In other words, the intersection $(z+W) \cap F(y)$ indeed contains a unique point $h_{z}(y)$ :

$$
h_{z}(y)=\sum_{i=0}^{k} \alpha_{i}(y) y_{i}=y_{0}+\sum_{i=1}^{k} \alpha_{i}(y)\left(y_{i}-y_{0}\right)
$$

To finish the proof, it is enough to show that $h_{z}(y)$ is continuous, that is, that each function $\alpha_{i}(y)$ is continuous for $0<i \leq k$.

Now, $z-\pi_{F}\left(y_{0}\right)=\sum_{i=1}^{k} \alpha_{i}(y) \pi_{F}\left(y_{i}-y_{0}\right)$ and the vectors $v_{i}=\pi_{F}\left(y_{i}-y_{0}\right)=$ $\pi_{F}\left(y_{i}\right)-\pi_{F}\left(y_{0}\right)$ are linearly independent for $i=1, \ldots, k$. So, we can choose the vectors $v_{i}, k<i \leq n$, such that the family $\beta=\left\{v_{i}: 0<i \leq n\right\}$ forms a basis for $\mathbb{R}^{n}$. Then, the numbers $\alpha_{i}(y)$ constitute the coordinates of $z-\pi_{F}\left(y_{0}\right)$ 
with respect to this basis $\beta$. Thus, $\left[\alpha_{1}(y) \cdots \alpha_{n}(y)\right]^{T}=A^{-1}\left(z-\pi_{F}\left(y_{0}\right)\right)^{T}$, where $A$ is the change of basis matrix which takes points in standard coordinates and gives their coordinates in the coordinate system induced by the basis $\beta$. (Thus, the $i$-th column of $A$ constitutes of the coordinates of $v_{i}$ with respect to the standard coordinate system.) Since all terms in $A^{-1}\left(z-\pi_{F}\left(y_{0}\right)\right)^{T}$ are continuous with respect to $y$, so are the functions $\alpha_{i}(y)$.

Lemma 5.7.6 For every compact $K \in \mathcal{J}_{k, n}$, there is an $\mathcal{F}_{k}$-continuous function $f: \mathbb{R}^{n} \rightarrow \mathbb{R}$ with $K=D(f)=\{x: \operatorname{osc}(f, x)=1\}$.

Proof. Let $\left\{\mathcal{L}_{i}: i<\omega\right\}$ be a sequence which justifies the inclusion of $K$ in $\mathcal{J}_{k, n}$. Let $E$ be a countable dense subset of $K$ and let $\left\langle p_{i}: i<\omega\right\rangle$ be a sequence of elements of $E$ enumerated so that every element of $E$ occurs in the sequence infinitely many times. We construct, by induction on $i<\omega$, a sequence $\left\langle D_{i} \subset \mathbb{R}^{n} \backslash K: i<\omega\right\rangle$ of disjoint closed balls of positive radius. For every $i<\omega$, there is a point $q_{i} \in B\left(p_{i}, 2^{-i}\right) \backslash\left(K \cup \cup \mathcal{L}_{i} \cup \bigcup_{j<i} D_{j}\right)$. We can find such a point because $K$ is nowhere dense and $p_{i} \notin \operatorname{int}\left(\bigcup \mathcal{L}_{i}\right)$. Choose $D_{i}$ to be a closed ball centered at $q_{i}$ and disjoint from $K \cup \cup \mathcal{L}_{i} \cup \bigcup_{j<i} D_{j}$. Let $f_{i}: \mathbb{R}^{n} \rightarrow \mathbb{R}$ be a continuous surjection vanishing identically outside of $D_{i}$. Now define $f=\sum_{i=1}^{\infty} f_{i}$. We claim $f$ is as desired.

The construction ensures that $\operatorname{osc}(f, p)=1$ if, and only if, $p \in K$ and that $\operatorname{osc}(f, p)=0$ otherwise. Since every element of $\mathcal{F}_{k}$ belongs to some $\mathcal{L}_{i}$, we may appeal to Lemma 2.3.1 to see that $f$ is $\mathcal{F}_{k}$ continuous.

The last of the lemmas is similar in character, purpose, and proof to $[4$, theorem 3.4]. 
Lemma 5.7.7 Let $f: \mathbb{R}^{n} \rightarrow \mathbb{R}$ be $\mathcal{F}_{k}$-continuous and let $N$ and $k$ be the natural numbers such that $n>k \geq \frac{n}{2}$. Then $K_{N}=\left\{p \in B[N]: \operatorname{osc}(f, p) \geq \frac{1}{N}\right\}$ belongs to $\mathcal{J}_{k, n}$.

Proof. For $j=1,2,3, \ldots$, let $\mathcal{L}_{j}=Z_{\frac{1}{j}, \frac{1}{4 N}}$, where we use $B=B[N+1]$ in the definition of $Z_{\frac{1}{j}, \frac{1}{4 N}}$. Let $K=K_{N}$. We claim that $\left\langle\mathcal{L}_{j}\right\rangle_{j}$ justifies the inclusion of $K \in \mathcal{J}_{n}$.

Clearly, $\mathcal{L}_{j} \subset \mathcal{L}_{j+1}$ and by Lemma 5.7.4, each $\mathcal{L}_{j}$ is closed. We also have $\bigcup_{j} \mathcal{L}_{j}=\mathcal{F}_{k}$ since for every $F \in \mathcal{F}_{k}$, the function $f \uparrow(F \cap B)$ is uniformly continuous. So, in order to finish, we need only show that $K \cap \operatorname{int}\left(\bigcup \mathcal{L}_{j}\right)=\emptyset$ for every $j$.

To see this, fix $j$ and $p \in B[N] \cap \operatorname{int}\left(\bigcup \mathcal{L}_{j}\right)$. Our proof will be complete if we can show $p \notin K$. To do this, we show that $\operatorname{osc}(f, p)<\frac{1}{N}$. This portion of the proof is quite similar to that of [4, theorem 3.4].

Let $F_{0}$ be the $k$-flat through $p$ parallel to the $k$-flat spanned by the first $k$ coordinate axes, and let $F_{1}$ be the $(n-k)$-flat through $p$ perpendicular to $F_{0}$. Since $f$ is $k$-continuous and $n-k \leq k$, the functions $f \uparrow F_{0}$ and $f \uparrow F_{1}$ are continuous. Hence, we can find a $\delta>0$ with $\delta<\frac{1}{2 j}$ so that if $r \in F_{0} \cup F_{1}$, and ||$r-p \|<\delta$, then $|f(r)-f(p)|<\frac{1}{4 N}$.

Decreasing $\delta$, if necessary, we can assume that $B(p, \delta) \subset B \cap \operatorname{int}\left(\bigcup \mathcal{L}_{j}\right)$. To finish the proof, it is enough to show that $|f(p)-f(q)|<\frac{1}{2 N}$ for every $q \in B(p, \delta)$, since then the oscillation of $f$ at $p$ will be less than $\frac{1}{N}$, so that $p \notin K$.

So, fix a $q \in B(p, \delta) \subset \bigcup \mathcal{L}_{j}$. Then, there exists a $k$-flat $F \in \mathcal{L}_{j}$ containing q. Since the convex hull of $F_{0} \cup F_{1}$ equals $\mathbb{R}^{n}$, there are $r_{0} \in F_{0} \cap F, r_{1} \in F_{1} \cap F$, and $\alpha_{0}, \alpha_{1} \in[0,1]$ such that $q=\alpha_{0} r_{0}+\alpha_{1} r_{1}$ and $\alpha_{0}+\alpha_{1}=1$. Notice that 
either $\left\|r_{0}-p\right\|<\delta$ or $\left\|r_{1}-p\right\|<\delta$, since otherwise

$$
\|q-p\|=\left\|\alpha_{0}\left(r_{0}-p\right)+\alpha_{1}\left(r_{1}-p\right)\right\| \geq \alpha_{0}\left\|r_{0}-p\right\|+\alpha_{1}\left\|r_{1}-p\right\| \geq \delta,
$$

contradicting the choice of $q$. Choose $r \in\left\{r_{0}, r_{1}\right\} \subset F_{0} \cap F_{1}$ with $\|r-p\|<\delta$. Then, $|f(p)-f(r)|<\frac{1}{4 N}$. Moreover, $\|r-q\|<2 \delta<\frac{1}{j}$ and $r, q \in F \in \mathcal{L}_{j}=$ $Z_{\frac{1}{j}, \frac{1}{4 N}}$. So, $|f(r)-f(q)| \leq \omega\left(f \uparrow(F \cap B), \frac{1}{j}\right) \leq \frac{1}{4 N}$. Therefore,

$$
|f(p)-f(q)| \leq|f(p)-f(r)|+|f(r)-f(q)|<\frac{1}{4 N}+\frac{1}{4 N}=\frac{1}{2 N}
$$

finishing the proof.

Proof of Theorem 5.4.3. If $D=D(f)$ for some $\mathcal{F}_{k}$-continuous function $f: \mathbb{R}^{n} \rightarrow \mathbb{R}$, then $D=\bigcup_{0<i<\omega} K_{i}$ where $K_{i}=\left\{p \in B[i]: \operatorname{osc}(f, p) \geq \frac{1}{i}\right\}$. So, by Lemma 5.7 .7 , each of these $K_{i}$ belong to $\mathcal{J}_{k, n}$.

Conversely, assume that $D=\bigcup_{i<\omega} K_{i}$, where each $K_{i}$ is compact and belongs to $\mathcal{J}_{k, n}$. Then, by Lemma 5.7.6, for every $i<\omega$ there exists an $\mathcal{F}_{k^{-}}$-continuous function $f_{i}: \mathbb{R}^{n} \rightarrow[0,1]$ with $D\left(f_{i}\right)=K_{i}$ and $\operatorname{osc}\left(f_{i}, p\right)=1$ for all points $p \in K_{i}$. Then, the function $f=\sum_{i<\omega} 3^{-i} f_{i}$ is $\mathcal{F}_{k}$-continuous and $D(f)=D$.

Our proof of Theorem 5.4.3 does not work for $k<\frac{n}{2}$. In fact, our proof of Lemma 5.7.4 depends heavily on the fact that $f$ is continuous on $(n-k)$-flats. In particular, the following example shows, that the conclusion of the lemma may be false for $k<\frac{n}{2}$.

Example 5.7.8 Let $g: \mathbb{R}^{2} \rightarrow \mathbb{R}$ be linearly continuous such that $g(0,0)=1$, while there exists a sequence $\left\langle p_{i} \in \mathbb{R}^{2}\right\rangle_{i<\omega}$ converging to $\langle 0,0\rangle$ such that 
$g\left(p_{i}\right)=0$ for all $i<\omega$. For example, if $f$ is given by (1.2), then $g(x, y)=$ $1-f(x, y)$ has this property. Define $h: \mathbb{R}^{3} \rightarrow \mathbb{R}$ as $h(x, y, z)=z g(x, y)$. Then $h$ is clearly linearly (so, $\mathcal{F}_{1^{-}}$) continuous. However, the set $Z_{2,1}$ for this function is not closed, as it contains all vertical lines through points $p_{i}$, but it does not contain their limit, the z-axis.

While this example does not preclude the existence of a version of Theorem 5.4.3 that would work for $k<\frac{n}{2}$, it emphasizes the difficulties.

An inspection of our the results presented in Section 5.7 yields some information about the structure of the sets in $\mathcal{D}_{k, n}, k \geq \frac{n}{2}$.

Corollary 5.7.9 If $n$ and $k$ are integers with $k \geq \frac{n}{2}$, the sets $\mathcal{J}_{k, n}$ are ideals.

Proof. Clearly any subset of a set $S \in \mathcal{J}_{k, n}$ also belongs to $\mathcal{J}_{k, n}$. We need only show that if $K_{1}$ and $K_{2}$ are elements of $\mathcal{J}_{k, n}$, then $K_{1} \cup K_{2} \in \mathcal{J}_{k, n}$. By Fact 5.7.3, we may assume that $K_{1}$ and $K_{2}$ are compact. Hence, by Theorem 5.4.3, there are $\mathcal{F}_{k}$-continuous functions $f_{1}, f_{2}: \mathbb{R}^{n} \rightarrow[0,1]$ such that $D\left(f_{i}\right)=K_{i}=\left\{z: \operatorname{osc}\left(f_{i}, z\right)=1\right\}$. Then the function $f_{1}+\frac{1}{2} f_{2}$ is $\mathcal{F}_{k^{-}}$ continuous and $D(f)=K_{1} \cup K_{2}$. Then, by applying Theorem 5.4.3 again, we see that $K_{1} \cup K_{2} \in \mathcal{J}_{k, n}$.

Although we are unable to characterize the sets $\mathcal{D}_{k, n}$ for all $k<n$, we are able to derive a sufficient condition for membership in $\mathcal{D}_{k, n}$. In particular, the following theorem gives us a tool for constructing discontinuity sets of $\mathcal{F}_{k}$-continuous functions without explicitly constructing the functions themselves.

Theorem 5.7.10 If $S$ is a countable union of compact members of $\mathcal{J}_{k, n}$, then $S \in \mathcal{D}_{k, n}$. 
Proof. Note that the "sufficiency" part of our proof of Theorem 5.4.3 depended only upon Lemma 5.7.6 which holds regards of $k$ and $n$.

\subsection{A Note on Baire Class}

One of the most well known results on separately continuous functions is Lebesgue's theorem that every separately continuous function $f: \mathbb{R}^{n} \rightarrow \mathbb{R}$ is of the $(n-1)$-st Baire class. We note that this result can be extended, with little effort, to the $\mathcal{F}_{k}^{+}$-continuous functions.

Proposition 5.8.1 Let $f: \mathbb{R}^{n} \rightarrow \mathbb{R}$ be an $\mathcal{F}_{k}^{+}$-continuous function. If $m=$ $\left\lceil\frac{n}{k}\right\rceil$, then $f$ is, at most, of the $(m-1)^{\text {st }}$ Baire class.

Proof. Let $r=n \bmod k$. Then we may write

$$
\mathbb{R}^{n}=\underbrace{\mathbb{R}^{k} \times \mathbb{R}^{k} \times \ldots \mathbb{R}^{k}}_{\left\lfloor\frac{n}{k}\right\rfloor} \times \mathbb{R}^{r}
$$

Where, of course, if $k \mid n$, we neglect the trivial factor $\mathbb{R}^{0}$. Hence, we may view $f$ as separately continuous in each of its $m$ variables. By Lemma 1.3.2, $f$ is of Baire class, at most, $m-1$.

In order to prove a partial converse, we first mention the result due to Maslyuchenko, Mykhailyuk, and Sobchuk (see [32]).

Theorem 5.8.2 If $X$ is any metric space and $f: X \rightarrow \mathbb{R}$ is of the $(n-1)^{\text {st }}$ Baire class, then there exists a separately continuous function $\phi: X^{n} \rightarrow \mathbb{R}$ such that $\phi(x, x, \ldots, x)=f(x)$ for all $x \in X$. 
From this, our partial converse follows immediately.

Proposition 5.8.3 If $f: \mathbb{R}^{k}$ is of Baire class $(m-1)$, then there exists a $\mathcal{F}_{k}^{+}$-continuous function $\phi:\left(\mathbb{R}^{k}\right)^{n} \rightarrow \mathbb{R}$ such that $\phi(x, x, \ldots, x)=f(x)$ for all $x \in \mathbb{R}^{k}$.

In the case that $k \not n$, the concept of "diagonal" is undefined, preventing us from extending Proposition 5.8.3 to this case. 


\section{Chapter 6}

\section{Open Questions}

1. For any function $f: X \rightarrow Y$, there is a maximal collection of sets $\mathcal{S}$ so that $f \in \mathcal{C}_{\mathcal{S}}$. What does this set tell us about the function $f$ ? In particular, is there a relationship between the "size" (in any sense) of $\mathcal{S}$ and the "size" (again, in any sense) of the set of discontinuities of $f$ ?

2. The question of characterizing the sets of discontinuities of $\mathfrak{C}\left(\mathcal{D}^{2}\right)$ functions has not been settled, neither has the question of characterizing the sets of discontinuities of linearly continuous functions defined on $\mathbb{R}^{n}$ where $n>2$ or many classes of $k$-flat continuous functions.

3. An understanding of how restriction continuities interrelate to the other classes of generalized continuities, i.e., within the class of separately continuous functions on $\mathbb{R}^{n}$, are there Darboux functions which are not connectivity? For some background on this question, see [12], and the references therein.

4. Are there linearly continuous function which are not of Baire class 1? 
Note that if such functions exist for some $\mathbb{R}^{n}$, then $n$ must be greater than 2 because of Lebesgue's theorem on the Baire class of separately continuous functions.

5. What is the size of $D(f)$ for typical linearly continuous functions? $\mathfrak{C}$ continuous functions for other classes of curves?

6. We proved in Chapter 4 that for every compact nowhere dense $K \subset$ $\mathbb{R}$ and every $\mathcal{C}^{2}$ function $\phi: \mathbb{R} \rightarrow \mathbb{R}$, that $\phi\left\lceil K \in \mathcal{D}_{1,2}\right.$. Does some version of this result hold for linearly continuous functions in higher dimensions? Perhaps for $\mathcal{F}_{k}$-continuous functions for some values of $k$ ?

7. Can our characterization of the discontinuity sets $\mathcal{D}_{k, n}\left(k \geq \frac{n}{k}\right)$ be made simpler or expressed in more straightforward terminology? 


\section{Bibliography}

[1] S. Agronsky, A. M. Bruckner, M. Laczkovich, and D. Preiss, Convexity conditions and intersections with smooth functions, Trans. Amer. Math. Soc. 285 (1985), 659-677.

[2] R. Baire, Sur les fonctions de variables reèlles, Ann. Mat. Pura Appli. 3 (1898), $1-122$.

[3] J. Boman, Differentiability of a function and of its compositions with functions of one variable, Math. Scand. 20 (1967), 249-268.

[4] J. C. Breckenridge and T. Nishiura, Partial Continuity, QuasiContinuity, and Baire Spaces, Bull. Inst. Math. Acad. Sincia 4 (1976), 191- 203.

[5] A. M. Bruckner, Differentiation of Real Functions, Lecture Notes in Mathematics 659, Springer-Verlag, New York, 1978.

[6] L. A. Cauchy, Cours D'analyse de l'Ecole Royale Polytechnique, Paris, 1821.

[7] K. Ciesielski, Set Theory for the Working Mathematician, London Math. Soc. Stud. Texts 39, Cambridge University Press, Cambridge, 1997. 
[8] K. Ciesielski, "Separately Continuous Functions on $\mathbb{R}^{n}$ : From Cauchy's Mistake through Lebesgue's Contributions, to Contemporary Results," submitted.

[9] K. Ciesielski and T. Glatzer, Functions Continuous on Twice Differentiable Curves, Discontinuous on Small Sets, Real Analysis Exchange, 37(2)(2012), 353-362.

[10] K. Ciesielski and T. Glatzer, Sets of Discontinuities of Linearly Continuous Functions, Real Analysis Exchange, 38(2), 337-389.

[11] K. Ciesielski and T. Glatzer, On Functions Continuous on Flats, accepted by Real Analysis Exchange. To appear.

[12] K. Ciesielski, J. Jastrzębski, Darboux-like functions within the classes of Baire one, Baire two, and additive functions, Topology Applications 103(2) (2000), 203-219.

[13] K. Ciesielski, L. Larson, and K. Ostaszewski, I-density continuous functions., Memoirs of the Amer. Math. Soc. 107 no.515, 1994.

[14] K. C. Ciesielski and T. Nishura, Continuous and smooth images of sets, Real Analysis Exchange, 37(2)(2012), 305-314.

[15] K. Ciesielski and J. Pawlikowski, Covering Property Axiom, CPA. A combinatorial core of the iterated perfect set model, Cambridge Tracts in Mathematics 164, Cambridge Univ. Press, 2004.

[16] K. C. Ciesielski and J. Rosenblatt, Restricted Continuity and a Theorem of Luzin, submitted. 
[17] J. P. Dalbec, When does restricted continuity on continuous function graphs imply joint continuity?, Proc. Amer. Math. Soc. 118 (2) (1993), 669-674.

[18] R. Engelking, General Topology, Polish Scientific Publishers, Warsaw, 1977.

[19] L. C. Evans and R. F. Gariepy, Measure Theory and Fine Properties of Functions, CRC Press, Boca Raton, 1992.

[20] K. Falconer, The Geometry of Fractal Sets, Cambridge University Press, Cambridge, 1985.

[21] A. Genocchi and G. Peano, Calcolo differentiale e principii calcolo integrale, Torino 1884.

[22] B. Grünbaum Convex Polytopes, John Wiley and Sons, London and New York, 1967. Second Edition, Springer 2003.

[23] H. Hahn, Über Funktionen mehrer Veränderlichen die nach jeder einzelnen Veränderlichen stetig sind, Math Zeit. 4 (1919) 306-313.

[24] M. Jarnicki and P. Pflug, Directional Regularity vs. Joint Regularity, Notices of the AMS, 58(7), (2011), 896 -904.-100.

[25] R. Kershner, The Continuity of Functions of Many Variables, Transactions of the AMS, $\mathbf{5 3}$ (1943), 83

[26] A. B. Kharazishvili, Strange Functions of Real Analysis $2^{\text {nd }}$ ed., Chapman and Hall/CRC, Boca Raton, 2006. 
[27] E. M. Landis and I. M. Iaglom, Remembering A. S. Kronrod, Mathematical Intelligencer 24(1), (2002).

[28] S. R. Lay. Convex Sets and Their Applications John Wiley and Sons, 1982. Reprinted by Dover, 2007.

[29] H. Lebesgue, Sur l'approximation des fonctions, Bull. Sciences Math, 22 (1898).

[30] H. Lebesgue, Sur les fonctions representable analytiquement, J. Math. Pure Appl. 6 (1905) 139-216.

[31] E. Marczewski and C. Ryll-Nardzewski, Sur la measurabilite des fonctions de plusieurs variables, Ann. Soc. Polon. Math. 25 (1952), 145-154.

[32] V. K. Maslyuchenko, V. V. Mykhailyuk, and O. V. Sobchuk, Construction of a Separately Continuous Function of $n$ Variables with a Given Diagonal, Matematychni Studii 12:1 (1999), 101-107. In Ukrainian.

[33] J. Matoušek, Lectures on Discrete Geometry, Springer, Heidelberg, 2002.

[34] V. V. Mykhailyuk, Construction of Separately Continuous Functions with Given Restriction, Ukrainian Mathematics Journal 55:5 (2003), 866-872.

[35] J. C. Oxtoby, Measure and Category, Graduate Texts in Mathematics 2, Springer-Verlag, New York, 1971.

[36] Z. Piotrowski, Topics in Separate Vs. Joint Continuity, Unpublished work in progress. 
[37] Z. Piotrowski, "Separate and Joint Continuity," Real Analysis Exchange, 11(2) (1985-1986), 293-322.

[38] Z. Piotrowski, The Genesis of Separate Versus Joint Continuity, Tatra Mountains Math. Publ. 8 (1996), 113 - 126.

[39] Z. Piotrowski and R. Vallin, Separately Continuous Functions: Approximations, Extensions, and Restrictions, Int. J. of Math. Sci. 54 (2003), 3469-3477.

[40] A. Roberts and D. Varberg, Convex Functions, Academic Press, New York, 1973.

[41] A. Rosenthal, On the Continuity of Functions of Several Variables, Math. Zeitschr. 63 (1955), 31-38.

[42] H. Royden, Real Analysis, $3^{\text {rd }}$ ed. Prentice Hall, Englewood Cliffs, NJ, 1988.

[43] L. Scheefer, Theorie der Maxima und Minima einer Function von zwei Variabeln, Math. Ann. 35 (1890), 541 -567.

[44] E. E. Shnol', Functions of Two Variables Continuous Along Straight Lines, Mat. Zametiki 62 (1997) 306-311. In Russian. English translation available as Math Notes 62 (1997) 255 -259.

[45] S. G. Slobodnik, An expanding system of linearly closed sets, Mat. Zametki 19 (1976), 67-84. In Russian. English translation available as Math Notes 19 (1976) 39-48. 
[46] J. Thomae Abriß einer Theorie der complexen Funktionen und der Thetafunktionen einer Veränderlichen, Louis Nebert Verlag, Halle 1870; 2nd edition: 1873.

[47] W. H. Young and G. C. Young, Discontinuous functions continuous with respect to every straight line, Quart. J. Math. Oxford Series 41 (1910). 